\title{
Why leave a job half done? Recent progress in enzymatic deracemizations
}

\author{
Alba Díaz-Rodríguez, Iván Lavandera,* and Vicente Gotor* \\ Departamento de Química Orgánica e Inorgánica, Instituto Universitario de Biotecnología de Asturias, \\ University of Oviedo, C/ Julián Clavería 8, 33006 Oviedo, Spain \\ Tel: +34 985103452 or +34 985 103448; E-mail: lavanderaivan@uniovi.es or vgs@uniovi.es
}

\begin{abstract}
In recent years the usefulness of enzymatic systems to obtain a single stereoisomerically pure compound starting from a racemate has been expanded. Moreover, current advances in protein engineering, molecular biology and modeling tools are the basis to improve the catalytic properties of enzymes to face novel synthetic challenges. Also, medium engineering and novel immobilization methods of (bio)catalysts are enhancing the productivity of biocatalytic processes making them suitable for being scalable. The development of multienzymatic protocols and the combination of enzymes with other catalysts are providing a wide range of synthetic possibilities that are expanding the scope of these transformations even at industrial scale. Herein we will describe an overview of recent (chemo)enzymatic deracemizations, focusing on the strategy employed (dynamic kinetic resolution, stereoinversion, cyclic deracemization or enantioconvergent process), the type of substrate (e.g., alcohols, amines, carboxylic acid derivatives or carbonylic compounds), and the biocatalyst(s) used.
\end{abstract}

Keywords. Biocatalysis, Cyclic deracemization, Deracemization, Dynamic kinetic resolution, Enantioconvergent process, Enzymes, Organic synthesis, Stereoinversion 


\section{Introduction}

The concept of reaction efficiency for chemists and engineers has undergone substantial changes. While historically the success of a chemical process relied entirely upon the product yield, aspects such as waste generation and toxic properties of the chemicals used were neglected. Additionally, increasing environmental concerns of society arose after the publication of Anastas and Warner in 1998 about the principles of eco-friendly chemical processes, the so-called Green Chemistry. ${ }^{[1]}$ These principles considered safety matters associated with the manufacture, use and disposal of chemical products, and the efficient utilization of raw materials with minimization of waste production through implementation of catalytic processes. As a result, an efficient chemical process is turning into a procedure where minimal amounts of waste are produced while the product yield is maximized.

In this context, biocatalytic methods are gaining more relevance as they meet several requirements such as excellent selectivities under mild reaction conditions. ${ }^{[2]}$ While traditionally the use of enzymes was restricted to the selective modification of racemic mixtures in kinetic resolution (KR) protocols, researchers realized the potential of enzymatic methodologies to afford enantiomerically pure compounds circumventing the maximum $50 \%$ yield inherent to KRs. In particular, the employment of enzymatic desymmetrizations starting from prochiral derivatives has demonstrated an enormous potential and obviously has still an extreme significance in many interesting asymmetric processes. ${ }^{[3]}$ However, racemates are more readily available starting materials than prochiral substrates. Thus, the development of other catalytic enzymatic strategies that enable access to $100 \%$ theoretical yield of stereoisomerically enriched compounds starting from accessible racemic mixtures, has attracted many efforts since mid-90s due to the simpler isolation and purification techniques required. ${ }^{[4]}$ Collectively, these protocols can be encompassed in the so-called deracemization systems. Whereas the first non-enzymatic examples can be ascribed to enantioselective protonations, ${ }^{[5]}$ in the case of enzymatic-mediated processes, the bioreduction of $\alpha$-substituted ketones that can racemize under basic conditions are probably the first ones. ${ }^{[6]}$

Among the different protocols to deracemize a racemic mixture (Scheme 1), dynamic kinetic resolutions, deracemizations via stereoinversion, cyclic deracemizations and enantioconvergent processes can be mentioned. This review, mainly covering the period from 2011 onwards, describes the application of these methods to the synthesis of enantioenriched compounds where the key step is performed by at least one biocatalyst. For the coverage of earlier examples the reader is referred to previous excellent reviews and monographs. ${ }^{[7-13]}$ 


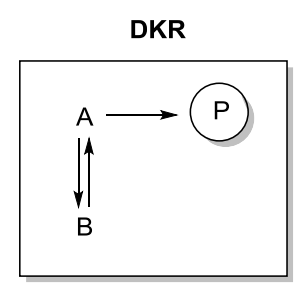

Cyclic deracemization

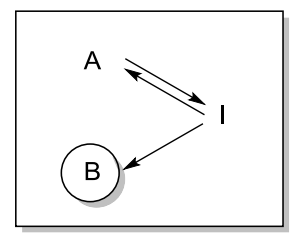

Stereoinversion

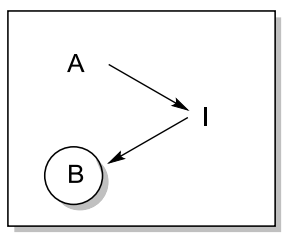

Enantioconvergence

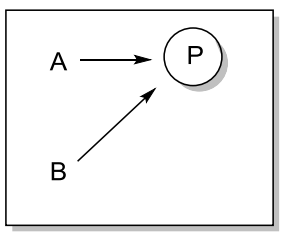

$\mathrm{A}, \mathrm{B}=$ enantiomers; $\mathrm{I}=$ intermediate; $\mathrm{P}=$ product

Scheme 1. General representations of deracemization strategies.

\section{Dynamic kinetic resolutions (DKRs)}

DKRs are by far the most employed strategies for deracemization. This approach relies on a racemization step of the substrate enantiomers combined with a selective transformation over one single enantiomer through a kinetic resolution process (Scheme 1). Theoretically, this would afford the final (enantiopure) product in quantitative yield. Noyori coined these systems as DKRs when performing Ru-catalyzed hydrogenations of $\alpha$-substituted $\beta$-keto esters. ${ }^{[14]}$ Due to the intrinsic selectivities shown by enzymes when reacting with racemic mixtures, the combination of biocatalyzed methodologies with different racemization techniques has allowed the efficient development of enzymatic-based DKRs. ${ }^{[15-19]}$

Among the different features that a good DKR process must accomplish, probably the most important is that the racemization reaction should be fast enough in order to maintain a perfect equilibration between both enantiomers in the racemic mixture. It must be taken into account that an inefficient rate would afford an increasing concentration of the non-reacting enantiomer and therefore, a loss in the selectivity within the time. In fact, ideally, racemization should be at least 10-times faster than the kinetic resolution. Reaction conditions for both processes must be compatible and should not interfere within each other, this is, the product formed must not racemize. One advantage of this method regarding classical KRs is that a reasonable selectivity $(E>20)$, can afford the final compound with high enantiomeric excess. ${ }^{[20]}$

Most of the DKR examples found in literature are related with the discrimination of racemic alcohols, amines, carbonylic and carboxylic acid derivatives, depending on the biocatalyst source. On the other hand, over racemization techniques, metal-mediated, the use of basic or acidic conditions, or the employment of reversible addition reactions, are the most prominent when combined with enzymes (vide infra). 


\subsection{DKRs over alcohol derivatives using hydrolases}

Due to the easy accessibility and readily racemization of alcohol compounds, many examples related to the deracemization of alcohol derivatives have recently been published. Thus, among the different ways to interconvert both alcohol antipodes, the use of a redox system which ensures the racemization through a carbonylic intermediate is probably one of the most commonly employed (Scheme 2a). ${ }^{[21]}$ Since Bäckvall's pioneering work combining a lipase with a metal-catalyst to resolve secondary alcohols via enantioselective acylation, ${ }^{[22]}$ research has been devoted to the employment of homogeneous metalcatalysts with hydrolases in order to undertake the resolution of valuable derivatives. The stability of these enzymes in organic solvents and the relatively low temperatures where these metallic complexes can work has greatly broadened the applicability of these systems. Considering the different metal catalysts, ruthenium complexes stand out as the most practical. For instance, Shvo's catalyst (1, Scheme $2 b$ ), ${ }^{[23]}$ has been recurrently chosen to achieve these transformations. Recently, Alcántara and co-workers have shown its use with lipase from Pseudomonas stutzeri (TL) to deracemize a series of benzoin derivatives in tetrahydrofuran (THF) at $50{ }^{\circ} \mathrm{C}$ in a sequential manner. ${ }^{[24]} \mathrm{In}$ a later study, the same authors reported that 2-methyltetrahydrofuran (2-MeTHF) was a more appropriate solvent to perform these DKRs in one-pot at $55{ }^{\circ} \mathrm{C} .{ }^{[25]}$ Bäckvall et al. also showed the application of $\mathbf{1}$ to racemize 3-substituted cyclic allylic alcohols in the presence of lipases from Candida antarctica type B (CAL-B) or Pseudomonas cepacia (PS-IM). Using this methodology, the corresponding $(R)$-acetates were obtained in usually high conversions (>60\%) and ee (up to $99 \%$ ). ${ }^{[2]}$ This DKR was performed in toluene at $60-80{ }^{\circ} \mathrm{C}$ as the reversible transfer hydrogenation of alcohols/ketones generally required high temperatures.

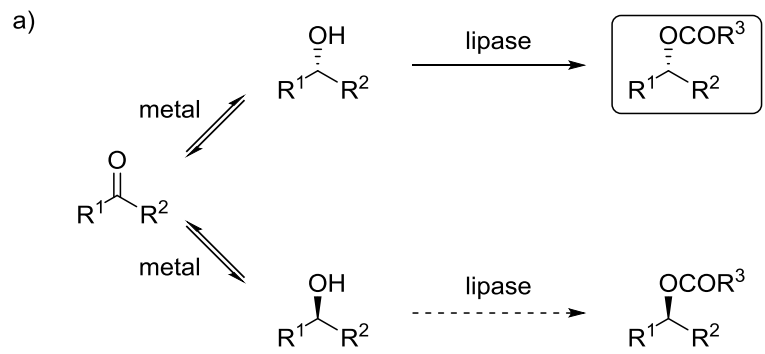

b)

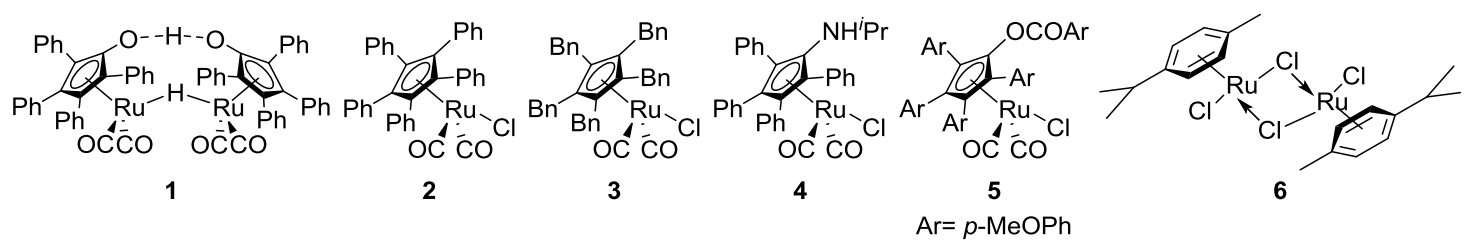

Scheme 2. a) Dynamic kinetic resolution of secondary alcohols through metal-based racemization via redox process. b) Examples of metal complexes used in these transformations.

However, in the last few years, other Ru-based complexes have been studied in order to address this issue. Thus, $\left(\eta^{5} \mathrm{C}_{5} \mathrm{Ph}_{5}\right) \mathrm{Ru}(\mathrm{CO})_{2} \mathrm{Cl}(2$, Scheme $2 \mathrm{~b})$, discovered by Bäckvall's group ${ }^{[27]}$ has proved to be an excellent catalyst working with different lipases at conditions close to room temperature. Among the different alcohols successfully resolved (Figure 1), a $\beta$-hydroxy nitrile precursor of duloxetine, ${ }^{[28]} \mathrm{N}$ protected piperidin-3-ols and pirrolidin-3-ols, ${ }^{[29]}$ cyclic allylic alcohols, ${ }^{[30]}$ homoallylic alcohols, ${ }^{[31]}$ and 1- 
tert-butoxypropan-2-ol, ${ }^{[32]}$ can be mentioned. With this complex, the addition of bases such as $t$-BuOK $(5 \% \mathrm{~mol})$ and $\mathrm{Na}_{2} \mathrm{CO}_{3}$ (1 equiv.) is necessary in order to activate it. Other ruthenium catalysts that have recently been employed in dynamic protocols are derivatives 3-6 (Scheme 2b). While 3 has been effectively utilized in the racemization of a series of aromatic and aliphatic secondary alcohols, ${ }^{[33]}$ catalyst

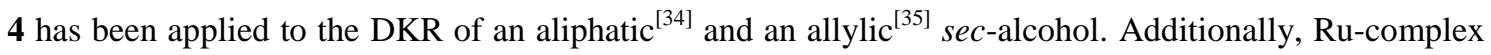
5 was used to synthesize enantioenriched 1,2-diarylethanols, ${ }^{[36]}$ and $\mathbf{6}$ has achieved excellent DKR protocols together with a salenol-derived ligand and TEMPO to deracemize 1-phenylethanol, ${ }^{[37]}$ or in combination with 1,4-bis(diphenylphosphino)butane as ligand in order to obtain enantioenriched benzoins (Figure 1). ${ }^{[38]}$ In all these processes, the addition of a base was also mandatory.

\section{Catalyst 2}

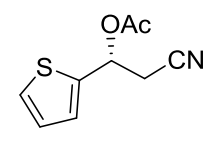

CAL-B

$87 \%, 98 \% e e^{[28]}$<smiles>CC(=O)O[C@H]1CCCN(Cc2ccccc2)C1</smiles>

PSL<smiles>CC(=O)OC1CCN(C(C)=O)C1</smiles>

PSL<smiles>CC(=O)O[C]1CCCCC1=Cc1ccccc1</smiles>

CAL-B<smiles>C=CC[C@H](OC(C)=O)c1ccccc1</smiles>

CAL-B

$91 \%, 96 \% \mathrm{ee}^{[29]} \quad 87 \%, 95 \% \mathrm{e} \mathrm{e}^{[29]} \quad 84 \%,>99 \% \mathrm{e} \mathrm{e}^{[30]} \quad 84 \%,>99 \% \mathrm{e} \mathrm{e}^{[31]}$

Catalyst 2<smiles>CC(=O)OC(C)COC(C)C</smiles>

CAL-B

$68 \%,>99 \%$ ee $e^{[32]}$
Catalyst $\underline{3}$<smiles>C[C](OC(C)=O)c1ccc(F)cc1</smiles>

CAL-B

$99 \%,>99 \%$ ee $[33]$

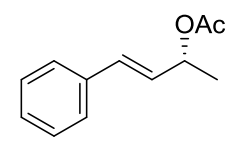

CAL-B

$99 \%, 98 \% e e^{[33]}$

\section{Catalvst 4}

CAL-B

$90 \%, 96 \% e^{[35]}$
Catalvst $\underline{5}$

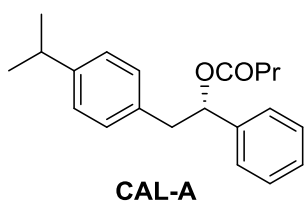

$84 \%, 94 \% e e^{[36]}$
Catalvst $\underline{6}$

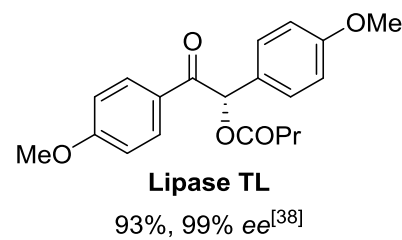

Figure 1. Examples of secondary alcohols deracemized via DKR using Ru-based complexes 2-6 as racemizing agents.

On the other hand, molecular biology tools have been used to modify biocatalysts improving their selectivities and/or stabilities. Thus, Bäckvall and co-workers have applied CAL-B mutants to access to a series of $(S)$-acetate products with excellent conversions and ee when combined with Ru-complex $\mathbf{2}$, inverting the selectivity displayed by the wild-type enzyme. ${ }^{[39]}$ A similar approach described by Ema $e t$ al. allowed the successful deracemization via DKR of several sterically hindered secondary alcohols using a mutant of lipase from Burkholderia (Pseudomonas) cepacia together with 2 to synthesize the corresponding $(R)$-esters in high yields $(>70 \%)$ and excellent selectivities $(>95 \%){ }^{[40]}$ A novel lipase preparation has also been described by Park and co-workers adding an ionic surfactant. In this case, lipase from B. cepacia, which is generally less active than CAL-B in these transformations, was coated with an ionic surfactant, showing an activity increase of even 2-3 folds against a series of aromatic alcohols. 
Moreover, under the presence of Ru catalysts $\mathbf{4}$ and $\mathbf{5}$, the DKR processes proceeded smoothly obtaining the $(R)$-acetylated derivatives in excellent yields $(>90 \%)$ and $e e(>95 \%) .^{[41,42]}$

Apart from ruthenium, other transition metals have been successfully employed to achieve chemoenzymatic DKRs. Ikariya and co-workers reported the use of $\left(\eta^{5} \mathrm{C}_{5} \mathrm{Me}_{5}\right) \operatorname{Ir}\left[\kappa^{2}(N, C)-\left(\mathrm{NHCMe}_{2}-2-\right.\right.$ $\mathrm{C}_{6} \mathrm{H}_{4}$ ), a bifunctional amidoiridium complex, combined with CAL-B in toluene at $30{ }^{\circ} \mathrm{C}$, obtaining the corresponding enantiopure $(R)$-acetates of several aliphatic and aromatic sec-alcohols in high yields $(>80 \%) .{ }^{[43]}$ More recently, Akai et al. have combined the use of an oxovanadium catalyst immobilized inside mesoporous silica (MPS), which ensured the compartmentalization between both metal- and biocatalysts (CAL-B or PS-IM). ${ }^{[4]}$ Thus, $(R)$-acetylated derivatives from a series of aromatic and allylic alcohols were synthesized at $35^{\circ} \mathrm{C}$ using heptane or acetonitrile (MeCN) as solvents in very high conversions $(>80 \%)$ and enantiomeric excess $(>95 \%)$. Moreover, when starting from compounds bearing the alcohol at benzylic position $(7$, Scheme 3$)$, the final $(R)$-acetate derivatives 8 could be attained as the sole products. In this case, the racemization proceeded through 1,3-transposition of the hydroxyl group.

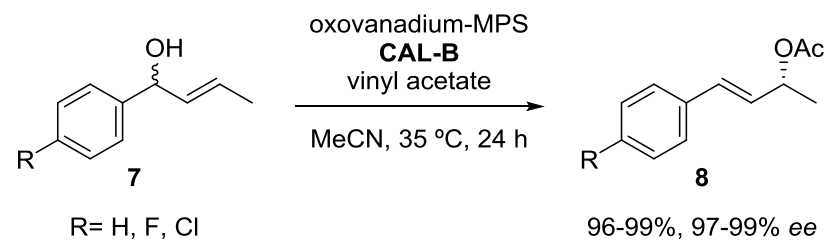

Scheme 3. DKR of allylic alcohols 7 through immobilized oxovanadium racemization giving access to acetates $(R)-\mathbf{8}^{[44]}$

The racemization of secondary alcohols can also be achieved in acidic conditions through the corresponding carbocation intermediate. Since aromatic alcohols are prone to form benzylic carbocations, many examples have recently been reported. To reach this goal different materials such as zeolites and cation-exchange resins have been employed in combination with different lipases. Thus, H- $\beta$ nanozeolite microspheres in combination with CAL-B,${ }^{[45]}$ resin Nafion SAC-13 together with CAL-B supported on ionic liquid-like phases under microwave conditions, ${ }^{[46]}$ and a nano solid super acid formed by $\mathrm{TiO}_{2} / \mathrm{SO}_{4}{ }^{-2}$ combined with CAL-B ${ }^{[47]}$ have successfully allowed the formation of various aromatic $(R)$-esters in high yields and $e e$ with a minimum formation of undesired by-products. A methodology proposed by Jaenicke et al. was even extended to non-aromatic derivatives such as 1-cyclohexylethanol or 2-pentanol. ${ }^{[48]}$ In this case an acidic $\beta$ zeolite was covered by an inert Silicalite-1 material to minimize secondary reactions such as product racemization or dehydration. In the presence of CAL-B in toluene at $60{ }^{\circ} \mathrm{C}$, the final $(R)$-esters were accessed in high conversions (>90\%) and selectivities (>99\%).

An especial case has been reported by Akai and co-workers ${ }^{[49]}$ which deracemized nitrone 9 using CAL-B and acyl donor 10 in $\mathrm{MeCN}$ at low temperature $\left(5-10^{\circ} \mathrm{C}\right)$, in order to synthesize tricyclic derivative 11 in a cascade protocol (Scheme 4a). This compound is an intermediate of (-)-rosmarinecine, which is precursor of natural alkaloids. After a detailed study, the authors proposed as the most plausible mechanism for racemization of $\mathbf{9}$ the elimination of the hydroxyl group after protonation, followed by the conjugate addition of water to the pyrrolinium salt $\mathbf{1 2}$ (Scheme 4b). 
a)

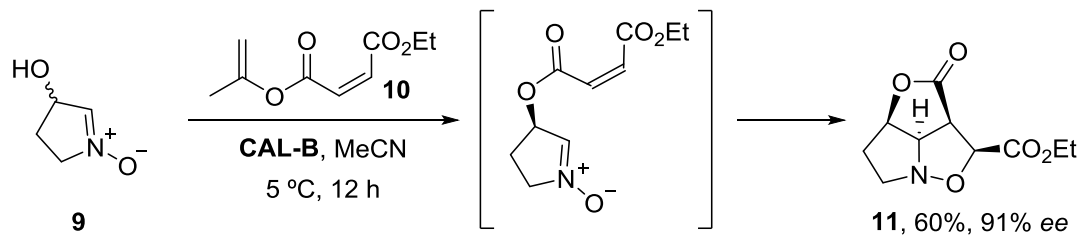

b)

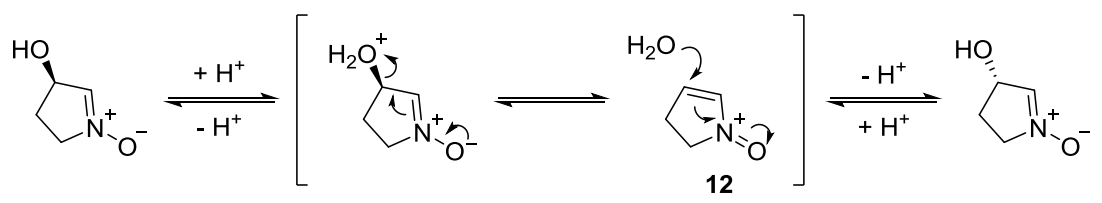

Scheme 4. a) DKR of nitrone 9 to obtain tricyclic derivative $\mathbf{1 1}$ through a cascade reaction. b) Plausible mechanism for racemization of compound 9. ${ }^{[49]}$

The use of a reversible addition reaction has been taken as an advantage to perform DKRs when the labile chiral intermediate obtained can be selectively (and irreversibly) transformed into the final enantioenriched product. Among the typical synthetic strategies that have been used for this purpose, the addition of cyanide, of a nitro or of a thiol derivative to an aldehyde, can be mentioned. Ramström and co-workers applied this concept to constitutional dynamic chemistry (CDC). ${ }^{[50]}$ This strategy is generally based on reversible interconnections between different molecules through (non-)covalent bonds, generating dynamic systems primarily under thermodynamic control. If the system is influenced by an external factor which preferentially selects one of the components, the dynamic system is forced to reequilibrate at the benefit of the selected species.

Thus, these authors reported the reaction between several aldehydes with acetone cyanohydrin to achieve the preparation of all possible cyanohydrin intermediates, which in the presence of immobilized Pseudomonas cepacia lipase (PSL-C-I) and an acyl donor, preferentially acylated one of the members of the library. ${ }^{[51]}$ In a later study, the reaction between several aldehydes and thiols led to a mixture of hemithioacetals which subsequently cyclized selectively by CAL-B-catalyzed intramolecular lactonization. ${ }^{[52]}$ Also, the simultaneous reaction of various aldehydes with 2-nitropropane and 1butanethiol to achieve a double parallel dynamic system, in which PS-IM preferentially acylated some of the intermediates, was developed. ${ }^{[53]}$ As an extension of these studies, the selective formation of a lamivudine precursor via dynamic addition-cyclization-acetylation cascade reaction mediated by subtilisin Carlsberg starting from two masked aldehydes (Scheme 5a), ${ }^{[5]}$ and the formation of enantioenriched 1,3-oxathiolan-5-one derivatives by DKR reaction between an aldehyde and a thiol plus intramolecular cyclization catalyzed by CAL-B (Scheme $5 \mathrm{~b}),{ }^{[55]}$ have recently been demonstrated. 
a)

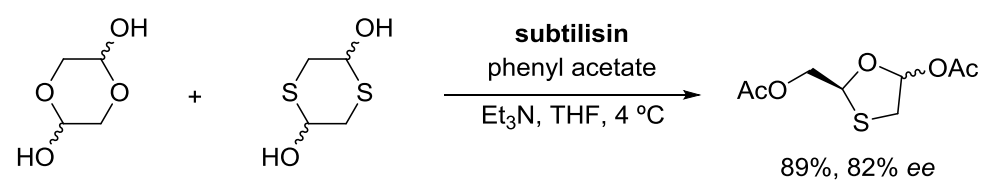

b)

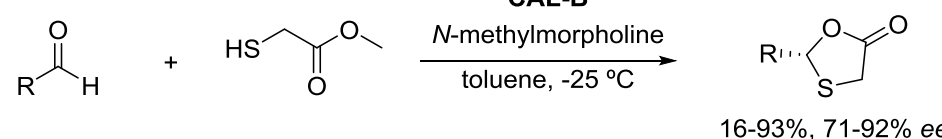

Scheme 5. a) Formation of a lamivudine precursor through a dynamic cascade protocol. ${ }^{[54]}$ b) DKR process to synthesize 1,3-oxathiolan-5-one derivatives. ${ }^{[55]}$

\subsection{DKRs over amino derivatives using hydrolases}

Again, among the different methodologies available to achieve the racemization of amines, the one which involves a metal-catalyzed redox process through the imine intermediate is the most commonly employed. When combined with a hydrolase-type enzyme, the corresponding enantiopure amides can be synthesized. Since Reetz described the metal-based DKR of 1-phenylethylamine using palladium over charcoal, ${ }^{[56]}$ different systems such as palladium mixtures, ruthenium, or iridium complexes, and $\mathrm{Ni}$ Raney have been demonstrated as good racemizing agents of amino derivatives. Due to the formation of the imine intermediate, the use of mild conditions is important in order to avoid secondary reactions such as hydrolysis into the ketone or dimerization. ${ }^{[17]}$

Pd-based racemization has widely been used in the last few years (Figure 2). Choi et al. explored the use of a $\mathrm{Pd}$ nanocatalyst entrapped in an $\mathrm{AlO}(\mathrm{OH})$ matrix at $70{ }^{\circ} \mathrm{C}$ together with a PSL-catalyzed $\mathrm{KR}$ in THF at room temperature in a one-pot sequential manner, in order to deracemize $\beta$-phenylalanine amide. Thus, after three cycles, the enantiopure $(R)$-amide was attained in $90 \%$ yield. ${ }^{[57]}$ In another example, Mexiletine, an antiarrhythmic agent, was resolved into an $(R)$-amide derivative $(71 \%$, 99\% ee) via DKR using CAL-B and Pd over charcoal in THF at $50{ }^{\circ} \mathrm{C}$ with $\mathrm{Et}_{3} \mathrm{~N}$ as base under 1 atm of $\mathrm{H}_{2}$. This amide was further polymerized with CAL-B to get access to a poly(amine-co-ester) bearing mexiletine molecules as pendant groups, serving as a potential prodrug candidate. ${ }^{[58]} \mathrm{Wu}$ and co-workers recently showed the synthesis of enantioenriched $(S)$ - $\alpha$-trifluoromethylated acetamides combining CAL-B and $\mathrm{Pd} / \mathrm{Al}_{2} \mathrm{O}_{3}$ as racemizing catalyst in toluene at $70{ }^{\circ} \mathrm{C}$ with molecular sieves. ${ }^{[59]}$ In some cases, the addition of an exogenous base improved the conversion, i.e. dimethylamine or triethylamine. Depending on the amine structure, different selectivities were attained, being especially high for 2,2,2-trifluoro-1phenylethylamine $(\mathrm{c} \sim 60-70 \%,>99 \% e e)$. When the reaction was carried out sequentially $(\mathrm{KR}+$ racemization $+\mathrm{KR})$, better conversions were observed. In a very recent report, ${ }^{[60]}$ the DKR of several aromatic amines was performed with CAL-B in toluene at $55^{\circ} \mathrm{C}$ under $\mathrm{H}_{2}(0.3 \mathrm{~atm})$, employing a $\mathrm{Pd} /$ layered double-hydroxide catalyst embedded with a surfactant (dodecyl sulfate anion) to modulate the surface acidity and specific surface area of the metal catalyst. Hence, very high conversions $(>95 \%)$ and $e e(>90 \%)$ were obtained for all tested substrates. 


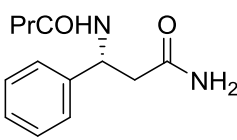

PSL

$90 \%,>99 \% e e^{[57]}$

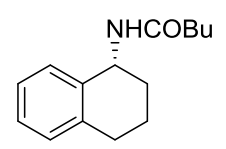

CAL-B

$99 \%,>99 \% e e^{[60]}$<smiles>Cc1cccc(C)c1OCC(C)NC(=O)CCN(CCO)CCO</smiles>

CAL-B

$71 \%, 99 \%$ ee $e^{[58]}$

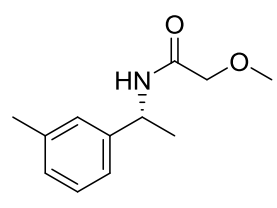

CAL-B

$87 \%, 99 \% e e^{[62]}$

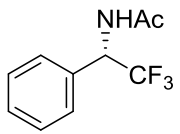

CAL-B

$68 \%, 99 \%$ ee $e^{[59]}$

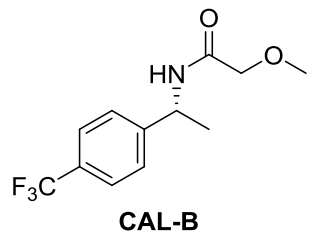

$89 \%, 97 \% e e^{[62]}$

Figure 2. Examples of primary amines deracemized via DKR using Pd complexes as racemizing agents.

Very recently, Bäckvall described the construction of an artificial metalloenzyme by co-immobilizing an enzyme and a metal on the same support, a siliceous mesocellular foam (MCF), through covalent attachment. The nanometallic component was responsible for the racemization of the amine $\left(\operatorname{Pd}^{0}\right)$, and the enzyme (CAL-B) for the KR. ${ }^{[61]}(R)$-Methoxyacetamide from 1-phenylethylamine was obtained with quantitative conversion and selectivity when the reaction was performed in toluene at $70{ }^{\circ} \mathrm{C}$ with $\mathrm{Na}_{2} \mathrm{CO}_{3}$ and molecular sieves under $\mathrm{H}_{2}(1 \mathrm{~atm})$. As an extension of this study, the palladium-supported catalyst over MCF was combined with two commercially available lipases (CAL-B and PS-C1), allowing the DKR process over a series of racemic aromatic amines affording the $(R)$-amides with high yields (>87\%) and excellent $e e(>97 \%) .{ }^{[62]}$ This metallic catalyst could be reused up to five times.

Although less employed, Ru-complexes can also be good candidates for racemizing amines. As a recent example, the use of Shvo's catalyst has been shown to deracemize 1-aryl- and 1-heteroarylpropan-2amines with CAL-B in toluene at $100{ }^{\circ} \mathrm{C}$ (yields $50-86 \%, e e>91 \%$ ). ${ }^{[63]}$

Secondary amines have also been resolved under dynamic conditions without the need of metals. Gotor and co-workers have described the deracemization of methyl 1,3-dihydro- $2 \mathrm{H}$-isoindole-1-carboxylate via enzymatic alkoxycarbonylation using PSL-C I in toluene or MTBE at $50-60{ }^{\circ} \mathrm{C}$ (Scheme 6a). Under these conditions, the substrate could racemize due to the high acidity of the proton at $\alpha$-position, obtaining the $(R)$-carbamate in high yields $(\sim 70 \%)$ and enantioselectivities $(90-95 \%){ }^{[64]}$ Liljeblad et al. described the DKR of $N$-tert-butoxycarbonyl-piperazine- and piperidine-2-carboxylic acid methyl esters through lipasecatalyzed acetylation. In this case, vinyl butanoate was used as acylating agent and the authors proposed that the acetaldehyde released in the acylation step could be responsible of the formation of an iminium salt (13), that could be deprotonated producing an achiral intermediate that could interconvert both enantiomers (Scheme 6b). Thus, using lipase A from Candida antarctica (CAL-A) immobilized on Celite in $\mathrm{MeCN}$ at $48{ }^{\circ} \mathrm{C}$, both secondary amines gave rise to the enantiopure $(S)$-amides in conversions around $60 \% .^{[65]}$ 
a)<smiles>CC(=O)[C@H]1NCc2ccccc21</smiles>

PSL-C I
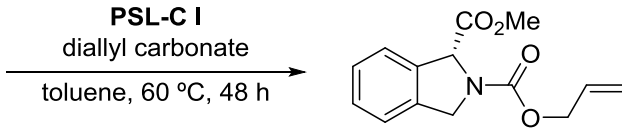

$72 \%, 94 \%$ ee

b)

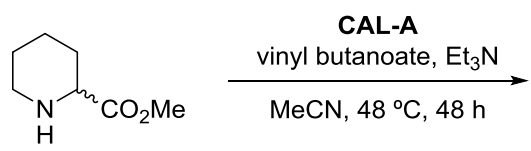

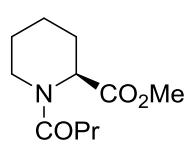

$60 \%,>99 \%$ ee

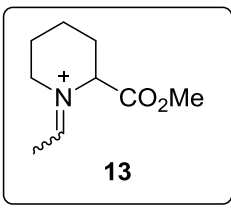

Scheme 6. Examples of DKRs over secondary amines through racemization: a) by an acidic proton abstraction; ${ }^{[64]}$ b) by the formation of a Schiff base. ${ }^{[65]}$

Ramström et al. have recently reported the preparation of a complex dynamic library arising from the simultaneous operation of different reversible processes such as imine formation/hydrolysis, transimination, hemithioacetal/hemithioaminal formation, and nitroaldol reactions, mixing different imines and aldehydes with a thiol and a nitro derivative. As a result, a complex mixture of compounds was generated, and in particular, two thiazolidinone compounds coming from an intramolecular acylation catalyzed by a lipase (CAL-B), were efficiently amplified. ${ }^{[66]}$

\subsection{DKRs over carboxylic acid derivatives}

\section{Using lipases and esterases}

Due to the electron-withdrawing character of carboxylic acid derivatives, the racemization of these compounds can be achieved via abstraction of the acidic $\alpha$-proton. Thus, most of the DKR examples with these derivatives when combined with lipase-catalyzed transformations are done under basic conditions. Recent examples are the CAL-B-catalyzed transesterification of oxazinones ${ }^{[67]}$ and azlactones ${ }^{[68]}$ using $\mathrm{Et}_{3} \mathrm{~N}$, the hydrolysis of ibuprofen methyl ester employing lipase from Candida rugosa (CRL) at basic $\mathrm{pH},{ }^{[69]}$ and the CAL-B-mediated hydrolysis of methyl 2,3-dihydrobenzo[b]furan-3-carboxylate derivatives in the presence of a diazaphosphorine base. ${ }^{[70]}$

Recently, another interesting approach was proposed by Wiggins and Bielawski, ${ }^{[71]}$ who employed ultrasonication to racemize poly(methylacrylate) chains with binol as pendant groups (Scheme 7a). Hence, hydrolysis catalyzed by cholesterol esterase in a biphasic system composed of methyl isobutyl ketone and phosphate buffer $(1: 1 \mathrm{v} / \mathrm{v})$, was combined with ultrasound pulses to produce the mechanicallyinduced isomerization of the unreacted $(R)$-isomer still attached to the polymer, affording $(S)$-binol.

Fox et al. have described the racemization of a cyclopropyl azlactone derivative by a spontaneous concerted [3,3]-sigmatropic rearrangement into an achiral dihydrooxepine intermediate (Scheme 7b). ${ }^{[72]}$ The azlactone was subsequently treated with CAL-B or PSL lipases in MTBE. Under transesterification conditions with EtOH, both enantiomers of a precursor of dehydrocoronamic acid, a building block of 
hepatitis $\mathrm{C}$ protease inhibitors, were obtained in excellent yields (>95\%) and moderate to excellent $e e$ [75\% for PSL $(1 R, 2 S), 95 \%$ for CAL-B $(1 S, 2 R)]$.

a)

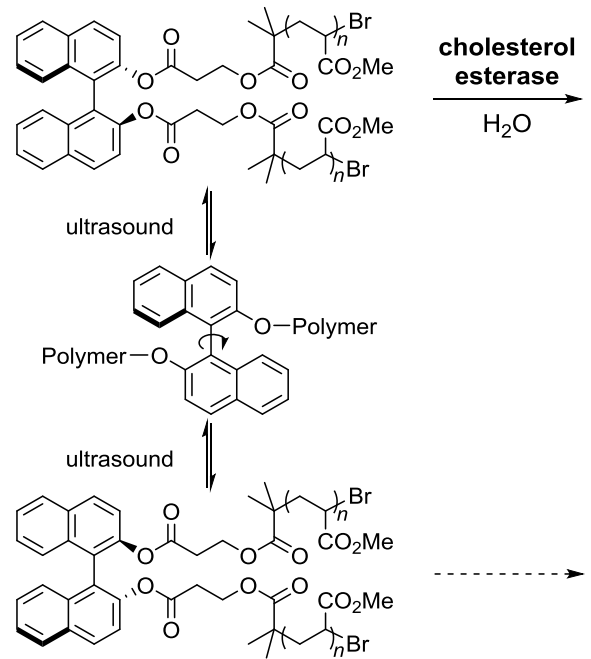

b)
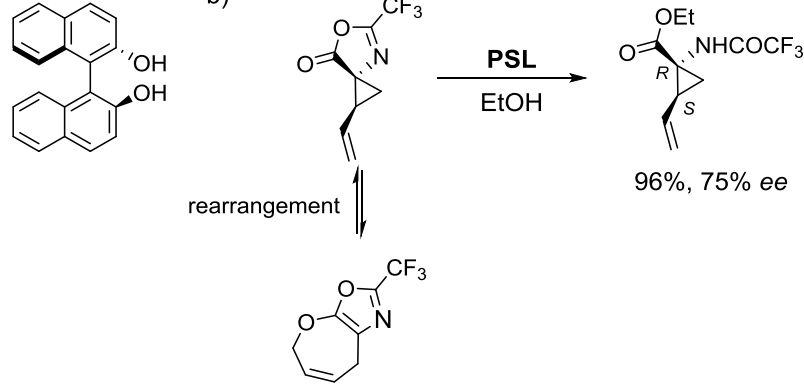

rearrangement

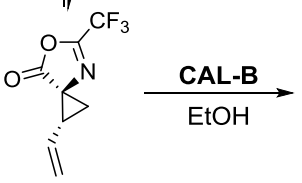
O 11 $97 \%, 95 \%$ ee

Scheme 7. Hydrolase-catalyzed DKRs to obtain: a) (S)-binol through ultrasound-based racemization; ${ }^{[71]}$ b) a dehydrocoronamic acid precursor through concerted [3,3]-sigmatropic rearrangement. ${ }^{[72]}$

Using proteases and acylases

Apart from lipases and esterases, other hydrolases have also been applied to dynamic protocols to synthesize valuable carboxylic acid compounds. Servi and co-workers have utilized $N$-Boc $\alpha$-amino thioesters as starting material to achieve DKR processes through selective hydrolysis, transesterification or aminolysis/ammonolysis catalyzed by different subtilisin preparations in the presence of bases. ${ }^{[73]}$ Trioctylamine was employed as the racemizing agent when the acidity of the $\alpha$-proton was high (e.g., aromatic derivatives), otherwise a stronger base such as 1,8-diazabicyclo-[5.4.0]undec-7-ene (DBU) was used (e.g., aliphatic derivatives). Thus, the corresponding enantiopure $N$-Boc $\alpha$-amino acids, esters or amides were attained in excellent yields at $37^{\circ} \mathrm{C}$ and in the presence of different nucleophiles. This methodology was further extended to synthesize naphthyl-derived amino acids. ${ }^{[74]}$

The use of racemases has also been described to perform the substrate racemization although their application is still limited due to their narrow substrate specificity and low stability in organic solvents. In fact, they are traditionally employed in processes involving amino acids, hydroxy acids and their derivatives. Recently, Campopiano and co-workers have mutated an $N$-acetyl amino acid racemase to improve its activity against $N$-acetyl-allylglycine, thus working at high substrate concentrations $(50 \mathrm{~g} / \mathrm{L})$, and when coupled to a D-acylase, D-allylglycine was obtained in Tris- $\mathrm{HCl}$ buffer at $40{ }^{\circ} \mathrm{C}$ in excellent yield $(89 \%)$ and $e e(>99 \%$, Scheme 8$) .{ }^{[75]}$ 


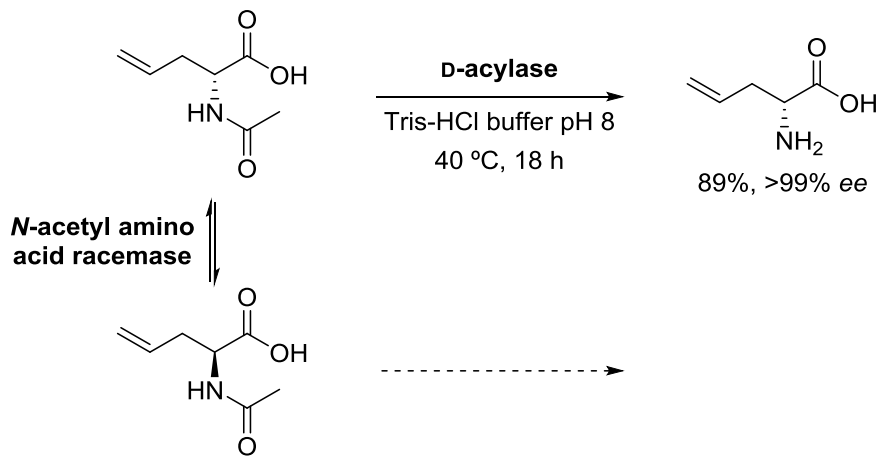

Scheme 8. Synthesis of D-allylglycine via DKR combining a racemase and a D-acylase. ${ }^{[75]}$

\section{Using nitrilases and amidases}

$\mathrm{Su}$ and co-workers have optimized the synthesis of D-phenylglycine and related derivatives starting from the corresponding phenylglycinonitriles, that racemize at basic $\mathrm{pH}$, using a recombinant nitrilase from Sphingomonas wittichii RW1. ${ }^{[76]}$ To avoid the decomposition of these substrates, a biphasic medium comprised of 1-octanol/buffer $(9: 1 \mathrm{v} / \mathrm{v})$ was utilized at $25^{\circ} \mathrm{C}$, obtaining the $\alpha$-amino acids in high yields $(60-80 \%)$ and $e e(90-95 \%)$. In a subsequent contribution, a cross-linked preparation of this nitrilase was able to achieve the deracemization of $N$-formyl-phenylglycinonitrile into $N$-D-formyl-phenylglycine $(95 \%$ yield and $97 \%$ ee ) in Tris- $\mathrm{HCl}$ buffer at $30{ }^{\circ} \mathrm{C}$. Moreover, this preparation could be recycled up to six times. ${ }^{[77]}$ Zheng et al. have reported the synthesis of $(R)$-o-chloromandelic acid, a precursor of Clopidogrel, a platelet aggregation inhibitor, via DKR hydrolyzing $o$-chloromandelonitrile with whole cells of overexpressed nitrilase from Labrenzia aggregata in a biphasic system toluene/phosphate buffer $(1: 9 \mathrm{v} / \mathrm{v})$ at $30{ }^{\circ} \mathrm{C} .{ }^{[78]}$ In this case the racemization proceeded through reversible addition of cyanide to $o$ chlorobenzaldehyde.

Amidases have also been applied to DKR processes. Yasukawa and Asano have showed the use of these enzymes coupled to racemases in order to synthesize several phenylalanine derivatives from the corresponding racemic phenylalanine amides. ${ }^{[79]}$ Hence, when a mutant from $\alpha$-amino- $\varepsilon$-caprolactam (ACL) racemase was co-expressed with D-amino acid amidase from Ochrobactrum anthropi SV3 in E. coli, the corresponding $(R)$-isomers were attained in high yields (70-80\%) and excellent $e e(>97 \%)$ in Tris- $\mathrm{HCl}$ buffer and $40{ }^{\circ} \mathrm{C}$. $(S)$-Phenylalanine was synthesized $(61 \%$ yield, $>99 \% e e)$ from the racemic amide precursor by co-expression of ACL racemase and L-amino acid amidase from Brevundimonas diminuta in $E$. coli in phosphate buffer and $40{ }^{\circ} \mathrm{C}$. Also, the deracemization of 2-amino-3phenylpropionitrile into $(R)$-phenylalanine (95\% yield, $90 \%$ ee) was performed, using a multienzymatic system composed by a purified non-stereoselective nitrile hydratase, together with the previously described E. coli co-expressing ACL racemase and D-amino acid amidase (Scheme 9). 


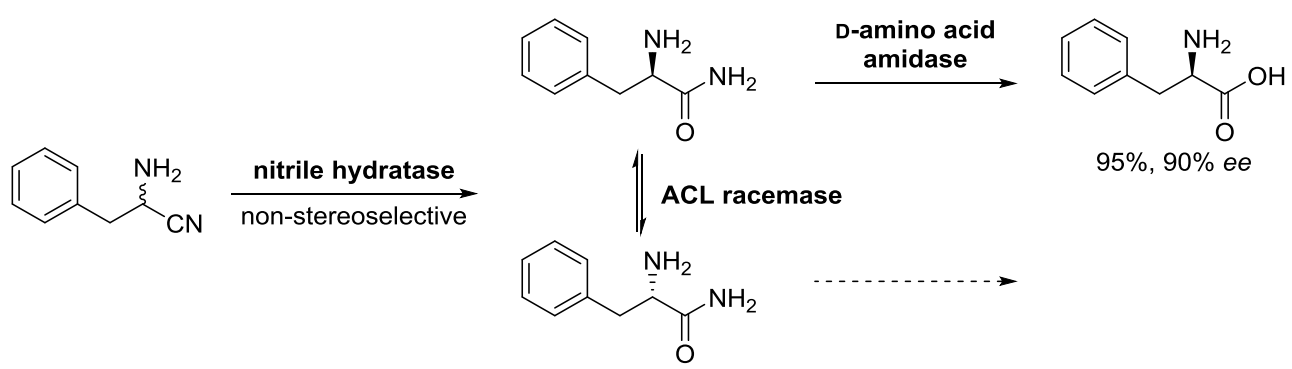

Scheme 9. Synthesis of $(R)$-phenylalanine from 2-amino-3-phenylpropionitrile combining a nitrile hydratase, a racemase and a D-amino acid amidase. ${ }^{[79]}$

\subsection{DKRs over carbonylic derivatives}

It is important to differentiate between the DKR of carboxylic acid derivatives and ketones or aldehydes as their reactivity is completely distinct. While for the first ones the biotransformation generally involves the interconversion of these species via hydrolysis, (trans)esterification or aminolysis, not creating additional chirality, for the second compounds the enzyme usually modifies the carbonyl group into a functional moiety, i.e. alcohol or amine, where a new chiral center is generated through a desymmetrization redox process. Therefore, in the latter case, it is very common to end up with diastereomeric mixtures (Dynamic Desymmetrizations). Obviously, these transformations present a major synthetic challenge than the previously mentioned DKR examples, since the enzyme must be both stereo(regarding the prochiral center) and enantio-selective (recognizing the chiral position).

Using alcohol dehydrogenases $(A D H s)$

Similarly, a dynamic process for $\alpha$-substituted carbonylic derivatives can be carried out under basic conditions taking advantage of the acidic character of the $\alpha$-proton. Hence, the bioreduction over the carbonyl moiety using an alcohol dehydrogenase is probably one of the most typical biocatalytic DKR systems, being described since 70 's. ${ }^{[6]}$ Recent examples include (Scheme 10a) the reduction of: i) $\alpha$ substituted $\beta$-keto esters with commercial and overexpressed ADHs, ${ }^{[80,81]}$ ii) $\alpha$-substituted 1,3 -diketones using commercial enzymes, ${ }^{[82]}$ iii) $\alpha$-substituted ketones employing overexpressed ADHs, ${ }^{[83]}$ and iv) $\alpha$ substituted aldehydes with an immobilized biocatalyst. ${ }^{[84]}$ In all cases a pH close to neutral was enough to develop an efficient racemization of the labile position adjacent to the carbonyl reacting group. 
a)<smiles>CC[C@H](C(=O)OC(C)C)[C@@H](C)O</smiles><smiles>CC(C)OC(=O)C(Cc1ccccc1)C(C)O</smiles>

RasADH

$78 \%,>99 \%$ de, $>99 \%$ ee $e^{[80]} 72 \%, 90 \%$ de, $>99 \%$ ee $e^{[80]}$<smiles>COC(=O)C(N)C(O)c1ccccc1</smiles>

commercial ADH<smiles>CCC(=O)C(C)C(C)O</smiles>

commercial ADH

$92 \%,>99 \%$ de, $>99 \%$ ee $[81] \quad 88 \%,>99 \%$ de, $>99 \%$ ee ${ }^{[82]}$<smiles>CC(O)C(C)c1ccccc1C#N</smiles>

ADH-A<smiles>N#Cc1ccccc1C1CC=CCC1O</smiles>

ADH-A

$93 \%,>99 \%$ de, $>99 \%$ ee $e^{[83]} 96 \%,>99 \%$ de, $>99 \%$ ee $e^{[83]}$

TeSADH= ADH from Thermoanaerobacter ethanolicus RasADH= ADH from Ralstonia sp.<smiles>CC(CO)c1ccccc1</smiles>

HLADH

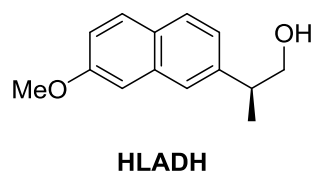

$79 \%,>99 \% e e^{[84]}$

$\mathrm{ADH}-\mathrm{A}=\mathrm{ADH}$ from Rhodococcus ruber $\mathrm{HLADH}=$ Horse liver ADH

b)

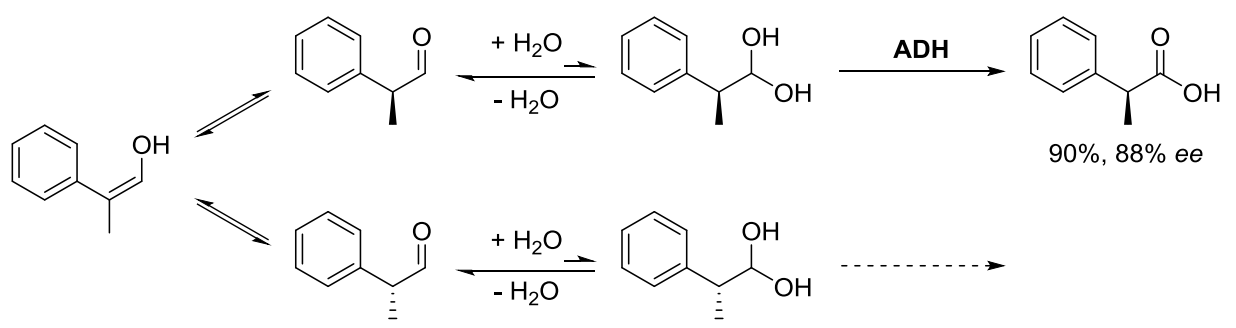

Scheme 10. a) Examples of derivatives obtained via bioreduction under dynamic conditions. b) DKR to produce $\alpha$-alkylated carboxylic acids by ADH-catalyzed oxidation of racemic aldehydes. ${ }^{[85]}$

Hollmann and co-workers recently showed the synthesis of $\alpha$-substituted carboxylic acids via ADHcatalyzed oxidation of the corresponding aldehydes (Scheme 10b). In this case, due to the racemization of these substrates at neutral-basic $\mathrm{pH}$, and based on the formation of the aldehyde hydrate, the authors envisaged a DKR to synthesize interesting derivatives such as 2-phenylpropionic acid. After enzymatic screening and reaction optimization, this compound could be obtained in a mixture of Tris-HCl buffer and dimethylsulfoxide (DMSO, 4:1 v/v) at $30^{\circ} \mathrm{C}$ in $90 \%$ yield and $88 \%$ ee $(S)$, showing that these enzymes can still offer uncommon, but advantageous, activities. ${ }^{[85]}$

\section{Using Baeyer-Villiger monooxygenases (BVMOs)}

In contrast to $\mathrm{ADHs}$, these oxygenases do not induce a novel chiral center as the catalyzed reaction is the formation of an ester from the corresponding carbonylic compound. Gotor and co-workers have reported the synthesis of acylated $\alpha$-hydroxy esters and secondary alcohols by DKR with BVMOs starting from $\alpha$ substituted $\beta$-keto esters and benzylic ketones, respectively (Scheme 11). These oxidoreductases are able to perform the selective Baeyer-Villiger oxidation of carbonylic compounds into the corresponding esters. In the first case phenylacetone monooxygenase (PAMO) from Thermobifida fusca and 4hydroxyacetophenone monooxygenase (HAPMO) from Pseudomonas fluorescens ACB showed the best results in terms of conversions $(>90 \%)$ and selectivities $[>99 \%(S)]$. The reactions were achieved in Tris$\mathrm{HCl}$ buffer with $\operatorname{MTBE}(5 \% \mathrm{v} / \mathrm{v})$ at $20-30{ }^{\circ} \mathrm{C} .{ }^{[86]}$ In the second example, a mutant from PAMO was employed over a series of racemic ketones in Tris- $\mathrm{HCl}$ buffer with $\mathrm{MeOH}(5 \% \mathrm{v} / \mathrm{v})$, and in the presence 
of a basic resin (Lewatit MP62) to facilitate the racemization process at $25^{\circ} \mathrm{C}$, affording the final products with moderate to very high conversions and $e e(60-90 \%) .{ }^{[87]}$
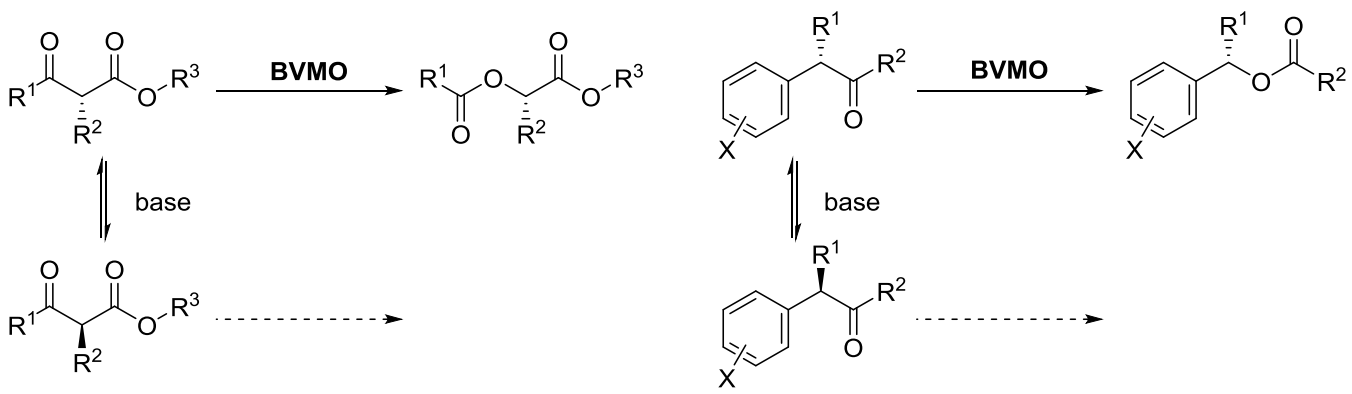

Scheme 11. Production of enantioenriched acylated $\alpha$-hydroxy acids and secondary alcohols via DKR using BVMOs under basic conditions. ${ }^{[86,87]}$

Using transaminases (TAs)

These biocatalysts are responsible of the transformation of carbonylic derivatives into the corresponding amines, therefore TAs have been utilized in dynamic protocols to synthesize enantioenriched amines (Figure 3). Thus, a number of relevant amines have been prepared such as $\alpha$-substituted $\beta$-amino esters, ${ }^{[88]}$ and a cyclic amino precursor of vernakalant, an antiarrhythmic agent. ${ }^{[89]}$ Very recently, Kroutil and coworkers have described the amination of a series of $\alpha$-substituted aromatic aldehydes to obtain chiral 2aryl-1-propylamines applying overexpressed TAs in E. coli in phosphate buffer at $30{ }^{\circ} \mathrm{C} .{ }^{[90]}$

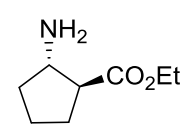

commercial TA

$58 \%, 98 \%$ de, $>99 \%$ ee ${ }^{[88]}$

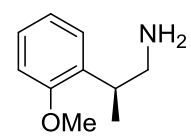

HN- $\omega$-TA

$50 \%, 99 \%$ ee ${ }^{[90]}$

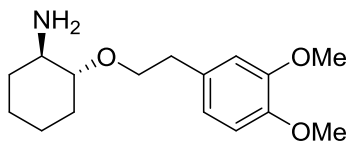

commercial TA

$81 \%,>99 \%$ de, $>99 \%$ ee ${ }^{[89]}$

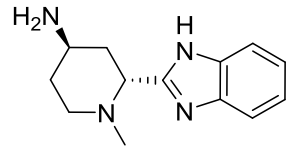

commercial TA

$85 \%,>90 \%$ de, $>99 \%$ ee $[91]$

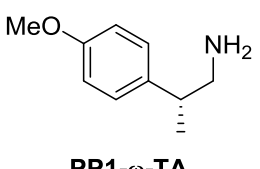

PP1- $\omega$-TA

$86 \%, 92 \%$ ee ${ }^{[90]}$

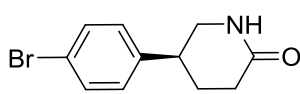

commercial TA

$84 \%,>99 \% e e^{[92]}$

PP1- $\omega-\mathrm{TA}=\mathrm{TA}$ from Pseudomonas putida $\quad \mathrm{HN}-\omega-\mathrm{TA}=\mathrm{TA}$ from Hyphomonas neptunium

Figure 3. Examples of derivatives obtained via enzymatic transaminations under dynamic conditions.

Peng et al. have developed the synthesis of a smoothened receptor inhibitor, used as target in some promising anticancer therapies, where the key step was the transamination of a 2-substituted 4-piperidone (last product, Figure 3). In this case the racemization proceeded through an achiral intermediate formed by a retro-aza-Michael/aza-Michael addition. Thus, the desired amine was obtained in a very high yield (>85\%), with excellent diastereo- (>90\%) and enantioselectivity (>99\%) using a commercial TA in a phosphate buffer-DMSO mixture $(4: 1 \mathrm{v} / \mathrm{v})$ at $50{ }^{\circ} \mathrm{C} .{ }^{[91]}$ Also, a lactam precursor of niraparib, an 
anticancer drug, was obtained through amination of a racemic aldehyde that intramolecularly cyclized in a cascade fashion. ${ }^{[92]}$

\section{Stereoinversions}

This technology, which consists in obtaining $100 \%$ of one of the substrate enantiomers as the sole product by inversion of the other enantiomer (Scheme 1), was traditionally linked to the preparation of optically pure alcohols although it has also been extended to the synthesis of enantioenriched amines and amino alcohols. ${ }^{[93]}$ In a stereoinversion process, a single enantiomer of the racemate is transformed into an achiral intermediate which is then irreversibly transformed into the opposite enantiomer. Thus, this methodology represents an attractive approach as a consequence of the availability of the corresponding racemates and the ready presence of one enantiomer (half of the final product) in the reaction mixture.

Stereoinversion processes can be performed by a number of redox biocatalysts whether in purified or semipurified form, or as whole-cell biocatalysts. The main difficulty of such processes is finding conditions under which two or more (bio)catalysts or chemicals are compatible.

\subsection{Stereoinversions over alcohol derivatives using oxidoreductases}

Traditionally, these bioprocesses have been carried out using whole-cells. In fact, it is true that the employment of whole cells has many advantages since they do not require cofactor addition, are easy to handle, and no protein purification is needed. However, in some cases, the selectivity of these biotransformations cannot be completely controlled since an unknown number of enzymes can be potentially active. Thus, the outcome for the targeted transformation can be influenced not only by the strain or the substrate structure but also by the cell growth conditions, the presence of metabolites or potential inducers. Despite these important disadvantages, this technology has proved to be successful for stereoinversion strategies. In these systems, theoretically the enantioselective oxidation of one enantiomer affords the prochiral ketone $(50 \%)$ together with the remaining enantiopure alcohol $(50 \%)$ in a first kinetic resolution; in the second step, the stereoselective reduction of the ketone with a stereocomplementary oxidoreductase provides the desired enantiopure alcohol with $100 \%$ yield.

For instances, Metschnikowia koreensis was recently employed for the stereoinversion process of a number of aryl secondary alcohols and 1,2-diols. ${ }^{[94]}$ This transformation was stereoselective and consisted of the sequential oxidation of the $(R)$-alcohol and subsequent reduction of the ketone formed to the $(S)$ enantiomer in high conversion (>88\%) and excellent optical purity (>98\%) in aqueous medium at $30{ }^{\circ} \mathrm{C}$. In another recent contribution, the same authors have deracemized a naphthyl alcohol derivative using Candida parapsilosis whole cells. ${ }^{[95]}$ It is important to highlight that in general long reaction times are required for the deracemization of secondary alcohols when using whole cells, therefore many investigations have been focused on overcoming this limitation. Recently, Chadha and co-workers have reported whole cell-mediated deracemizations of this type of substrates employing C. parapsilosis ATCC 7330 in phosphate buffer at $25^{\circ} \mathrm{C} .{ }^{[96]}$ In this work, deracemization of 1-arylethanol derivatives and 4phenyl-2-butanol, resulted in the formation of the $(R)$-enantiomers in excellent optical purity (up to $99 \%$ 
$e e$ ) and good yields (up to 78\%) at short reaction times ( $3 \mathrm{~h}$ ) by tuning the growing conditions. In a subsequent work, it was observed that this system preferred deracemizing allylic alcohols with $E$ configuration rather than $Z .^{[97]}$

In some cases whole-cell biotransformations are not successful as the required cofactors are not efficiently regenerated in situ. This drawback was investigated and overcome using a recombinant $E$. coli bearing two pyridine nucleotide transhydrogenases PNTs (PntA and PntB), to rebalance the cofactor pools in the pathway catalyzed by two carbonyl reductases from Candida parapsilosis (SCR and RCR), improving the flux of $\mathrm{NAD}^{+}$(needed by RCR) into NADPH (needed by SCR). ${ }^{[98]}$ In this way, the efficient preparation of $(S)$-1-phenyl-1,2-ethanediol (95\% yield, 97\% ee) was achieved from the $(R)$ alcohol through sequential oxidation/reduction reactions by the stereocomplementary action of SCR and RCR (Scheme 12).

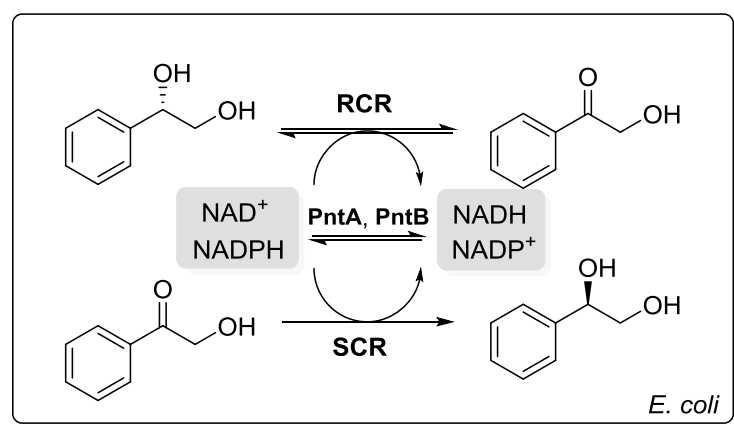

Scheme 12. Engineered E. coli harboring RCR, SCR, PntA and PntB for the production of (S)-1-phenyl-

$$
\text { 1,2-ethanediol. }{ }^{[98]}
$$

In another example, Alcántara and Molinari groups reported the deracemization of benzoins to obtain the (S)-enantiomers using Pichia glucozyma yeast. ${ }^{[99]}$ The use of a two-liquid phase system (phosphate buffer and $n$-heptane or isooctane), allowed the preparation of several $(S)$-benzoins avoiding the formation of the corresponding diols, favoring the isolation of the products and improving the enantiomeric excess (up to $99 \%)$.

An interesting methodology for the efficient one-pot deracemization of aromatic 2-hydroxyacids into the $(R)$-enantiomers (Scheme 13), was achieved combining resting cells of Pseudomonas aeruginosa CCTCC M 2011394 and Saccharomyces cerevisiae ZJB5074 with very good conversions (up to 99\%) and excellent $e e$ (up to $99 \%$ ) via tandem oxidation and reduction reactions in phosphate buffer at $30{ }^{\circ} \mathrm{C}$. ${ }^{[100]}$ The authors also demonstrated the applicability of this biotransformation in a $3-\mathrm{g}$ scale for the synthesis of a valuable precursor of an anti-thrombotic drug, Plavix, obtaining the desired $(R)$-hydroxy acid in $90 \%$ isolated yield and $>99 \% e e$. 


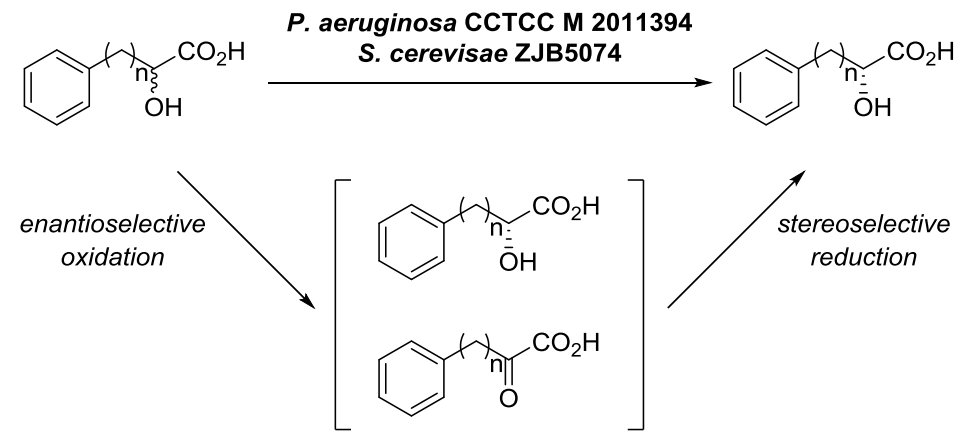

Scheme 13. Stereoinversion of the 2-hydroxyacid precursor of anti-thrombotic drug Plavix. ${ }^{[100]}$

The use of purified enzymes has dramatically enhanced the stereocontrol of such stereoinversion processes, as additional parameters can now be customized and adjusted to reach the desired outcome. Hence, the stereoinversion of secondary alcohols has widely been described using stereocomplementary alcohol dehydrogenases, which can selectively catalyze both oxidation and reduction reactions in a concurrent manner. ${ }^{[101]}$ A recent case where ADHs were employed in a concurrent manner was reported by our group. ${ }^{[102]}$ In this study, lyophilized or resting E. coli cells overexpressing ADH from Rhodococcus ruber DSM 44541 (E. coli/ADH-A) were used along with ADH from Lactobacillus brevis (LBADH) for the deracemisation of $s e c$-alcohols using 1-phenylethanol as model substrate, obtaining the $(R)$-alcohol with total conversion and $>99 \% e e$ in aqueous medium at $30{ }^{\circ} \mathrm{C}$. It is worth noting that no addition of cofactor was required for the first oxidative reaction and that the external NADPH-recycling system was the driving force of the process.

Laccases are $\mathrm{Cu}$-dependent oxidases that can be used together with electron mediators, and have shown great versatility in organic synthesis. In a recent example, the stereoinversion of a $\beta, \beta$-dihalogenated secalcohol was achieved via one-pot two-step protocol, performing first in a biphasic medium at $20^{\circ} \mathrm{C}$ the non-selective oxidation to the ketone intermediate catalyzed by laccase from Trametes versicolor and TEMPO, followed by the stereoselective bioreduction catalyzed by overexpressed $\mathrm{ADH}-\mathrm{A}$ at $30{ }^{\circ} \mathrm{C}$ (Scheme 14). ${ }^{[103]}$ The ADH-catalyzed bioreduction determined the ee of the process. Thus, the deracemization of 2,2-dichloro-1-phenylethanol was accomplished with excellent conversion and ee $(>97 \%)$.

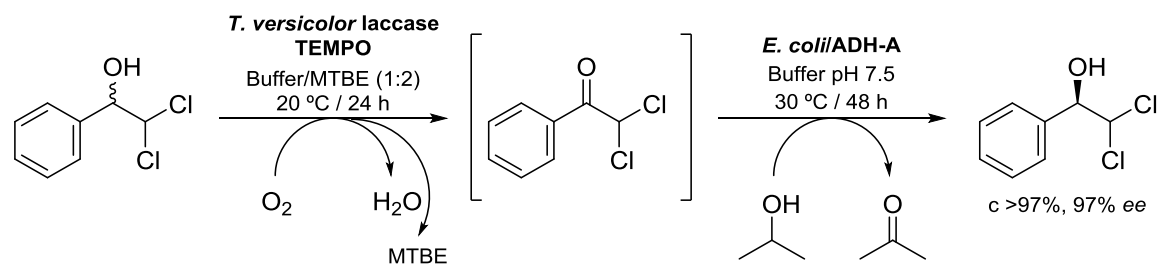

Scheme 14. Deracemization of 2,2-dichloro-1-phenylethanol using laccase/TEMPO and an alcohol dehydrogenase. ${ }^{[103]}$ 


\subsection{Stereoinversions over amino derivatives}

Using two transaminases

In the last few years, the number of deracemization examples of racemic amines employing two enantiocomplementary transaminases through a deamination/amination sequential reaction has increased. Ideally, in a first step the enantioselective oxidative deamination leads to the prochiral ketone (50\%) along with the remaining enantiopure amine (50\%); then, in the second step, the stereoselective amination of the ketone with a stereocomplementary $\omega$-TA gives access to the final chiral amine in $100 \%$ yield. The asymmetric synthesis of amines using this tandem protocol is advisable when the racemic amine is more accessible than the corresponding ketone, or when such ketone is unstable. Yun and co-workers have recently used this approach in a one-pot one-step fashion to deracemize aromatic primary amines, using (S)-selective $\omega$-TAs from Polaromonas sp. JS666 [(S)- $\omega$-TAPO] or Vibrio fluvialis JS17 [(S)-TAVF], and $(R)$-selective $\omega$-TAs from Mycobacterium vanbaalenii $[(R)$ - $\omega$-TAMV] or Neosartorya fischeri $[(R)-\omega$ TANF] (Scheme 15). ${ }^{[104]}$ This method enabled the synthesis of the $(R)$-antipodes in good conversions (70$99 \%$ ) and excellent $e e$ values (up to $>99 \%$ ). The key of this strategy was the use of an amino acceptor $(\alpha-$ ketoglutaric acid), that was not a good substrate for the second TA.

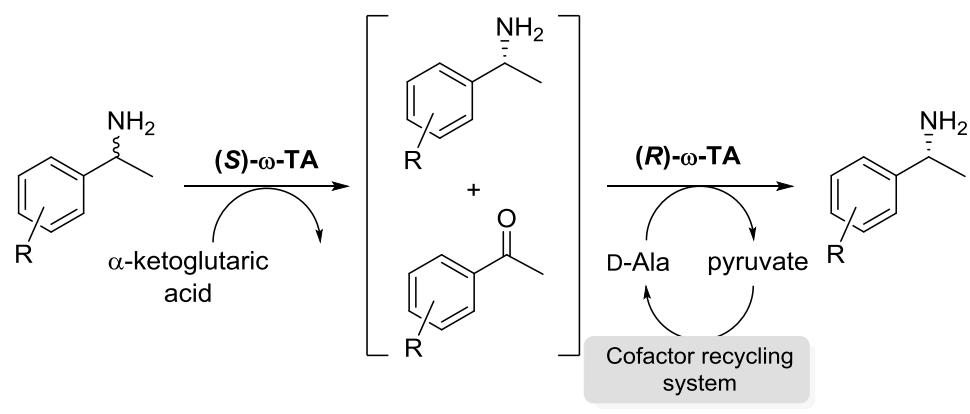

Scheme 15. Deracemization of racemic primary amines using two stereocomplementary TAs. ${ }^{[104]}$

\section{Using an oxidase and a dehydrogenase}

The feasibility of multienzymatic networks involving oxidases and dehydrogenases has efficiently afforded optically pure amino acids starting from the corresponding racemates. Patel et al. have applied this methodology to the synthesis of a key intermediate of a type II diabetes drug $[(S)-\mathbf{1 4}] .{ }^{[105]}$ First, a recombinant amino acid oxidase from Trigonopsis variabilis provided the enantioselective oxidation of the $(R)$-amino acid leaving the $(S)$-antipode intact (Scheme 16a). Then, this keto acid was transformed into the desired $(S)$-amino acid by means of a reductive amination using whole cells from Sporosarcina ureae SC16048 containing an $(S)$-amino acid dehydrogenase. The reaction was carried out at 1.95-g scale in aqueous medium at $28{ }^{\circ} \mathrm{C}$ yielding $(S)-\mathbf{1 4}$ in $54 \%$ overall yield and $>99 \% e e$. A two-step protocol was needed since the oxidase was otherwise inhibited. 
a)

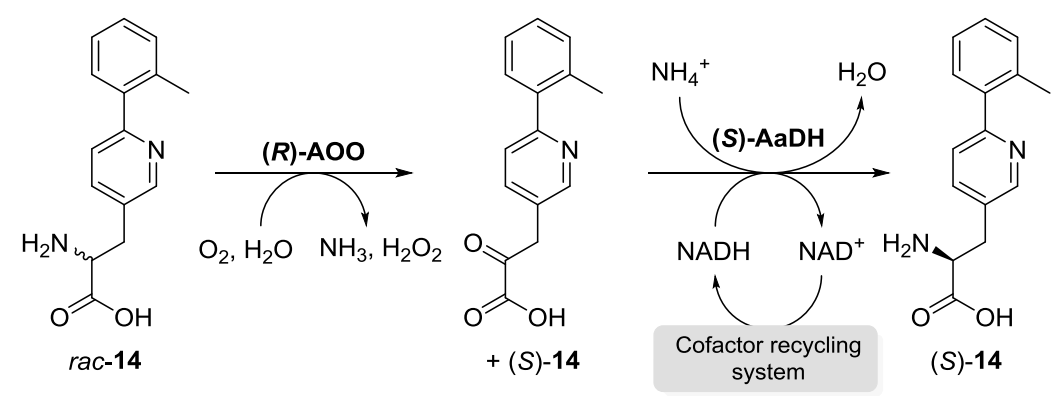

b)

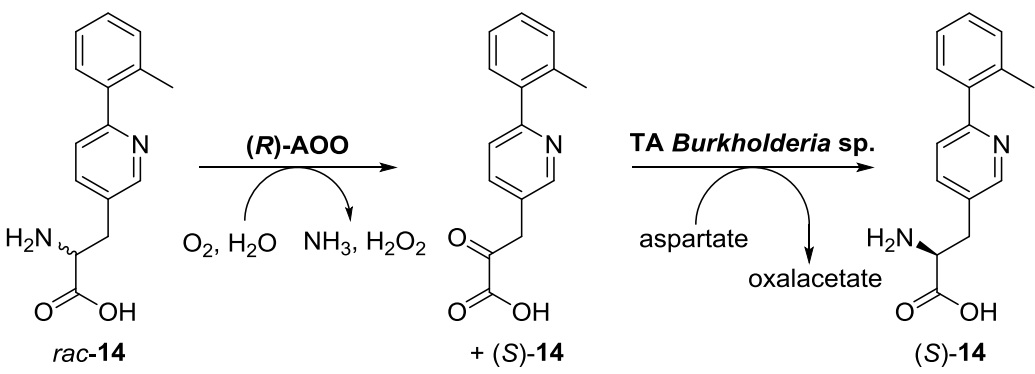

Scheme 16. Deracemization strategy of an amino acid combining: a) an amino acid oxidase and an amino acid dehydrogenase; b) an amino acid oxidase and a transaminase. ${ }^{[105]}$

Using an oxidase and a TA

Another related deracemization approach for the production of $(S)$-14 was based on the amino group oxidation to the corresponding keto derivative intermediate using the recombinant $(R)$-amino acid oxidase from $T$. variabilis, which was further aminated by a recombinant (S)-transaminase from Burkholderia sp. (Scheme 16b). ${ }^{[105]}$ Using this strategy, the one-pot biotransformation could be done in buffer at $30{ }^{\circ} \mathrm{C}$. The applicability of this multienzymatic approach was shown by scaling up this bioprocess at $607 \mathrm{~g}$ of the racemic amino acid to obtain the enantiopure $(S)$-amino acid in $66 \%$ yield and $>99 \%$ ee.

In another study, a non-proteinogenic amino acid such as L-homoalanine was employed as a model substrate in a similar approach. D-Amino oxidase from Rhodotorula gracilis (DAAO), a FAD dependent enzyme, was fused to Vitreoscilla hemoglobin $(\mathrm{VHb})$ to form VHb-DAAO. ${ }^{[106]}$ This protein improved the activity and stability of DAAO, and achieved the stereospecific oxidation of D-homoalanine to the corresponding imine, which was further hydrolyzed to form the keto acid intermediate. This biotransformation was then combined with $V$. fluvialis JS17 transaminase using benzylamine as the amino donor to obtain L-homoalanine. To avoid product inhibition, a biphasic system was necessary boosting the conversion of the process $(97 \%, e e>99 \%)$.

\section{Cyclic deracemizations}

Cyclic deracemization systems traditionally differ from stereoinversions in the use of a non-selective irreversible reaction combined with a second selective transformation that accumulates the enantiomer which is not transformed (Scheme 1). Hence, a typical deracemization example consists of a selective 
enzyme-catalyzed oxidation into a prochiral intermediate, which is then reduced back via non-selective chemical reduction process. ${ }^{[107]}$ Therefore, depending on the selectivity of the enzyme, after a certain number of cycles an enantiopure product can be reached.

\subsection{Cyclic deracemizations over alcohol derivatives}

In these processes, the enzymatic selective step can be performed with isolated enzymes or whole-cell biocatalysts. As a recent example, Kurina-Sanz et al. used undifferentiated plant cells from Gardenia jaminoides to deracemize 1-phenylethanol and other $s e c$-alcohol derivatives. ${ }^{[108]}$ This biocatalyst could oxidize the $(S)$-enantiomer from the racemic mixture leaving the other enantiomer untouched in a kinetic resolution. Then, $\mathrm{NaBH}_{4}$ was added for the reduction of the corresponding ketone. Although the activity of the cells was clearly affected by the reducing agent, when entrapping the biocatalyst in calcium alginate this strategy could be expanded to a series of secondary alcohols, and after several cycles the corresponding $(R)$-alcohols could be prepared in yields $>82 \%$ and $71-97 \%$ ee .

\subsection{Cyclic deracemizations over amino derivatives}

\section{Using an amino oxidase and a non-selective chemical reducing agent}

Another approach to obtain enantiopure amines is based on the combination of an amino oxidase with a chemical reagent. The enzyme performs the enantioselective oxidation of the racemic amine to afford the corresponding imine or iminium ion (depending on the substrate) coupled to a non-selective reducing agent, obtaining back the enantioenriched starting material. After several rounds, the accumulation of one enantiomer of the amine can be successfully realized. Recently, recombinant cells of E. coli overexpressing cyclohexylamine oxidase (CHAO) from Brevibacterium oxydans IH-35A, in combination with the borane-ammonia complex, were applied by Lau and co-workers to deracemize several primary amines into the corresponding $(R)$-enantiomers with moderate yields $(62-75 \%)$ and excellent $e e(>97 \%)$ in phosphate buffer at $30{ }^{\circ} \mathrm{C} .{ }^{[109]}$ In a later report, the same authors improved the performance of this biocatalyst by rational design. Five single-point mutants were prepared and tested in the same process, finding that the activity of some mutants was enhanced. ${ }^{[110]}$ A variant was used in the deracemization of 1-aminotetraline, precursor of Norsertraline, a selective serotonin reuptake inhibitor, yielding $(R)$-1aminotetraline in $76 \%$ and $>99 \%$ ee (Scheme 17). Recently, this strategy was extended to a secondary amine. ${ }^{[111]}$ Thus, the deracemization of 2-methyl-1,2,3,4-tetrahydroquinoline was achieved with a new discovered mutant of CHAO giving access to the $(R)$-amine in $76 \%$ yield and $>97 \%$ ee.

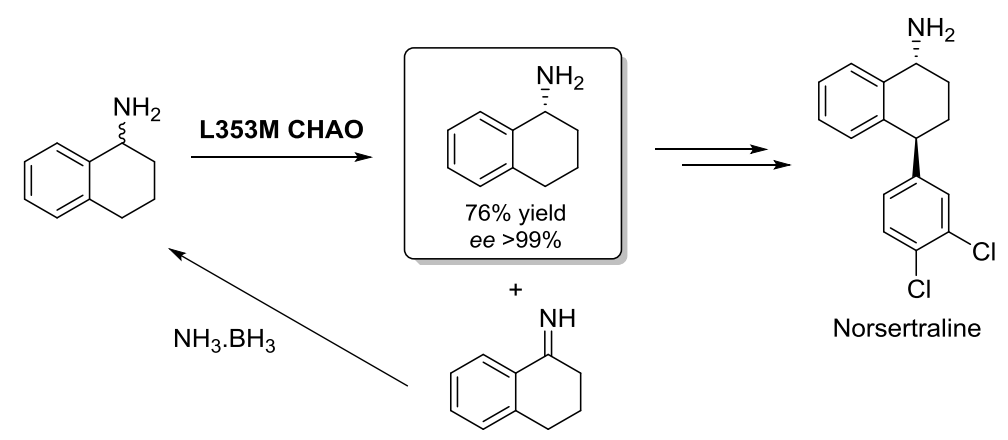

Scheme 17. Cyclic deracemization applied to the synthesis of a precursor of Norsertraline. ${ }^{[110]}$ 
In recent years, evolved variants of monoamine oxidase $\mathrm{N}$ from Aspergillus niger (MAO-N) have found new applications in the preparation of enantiopure amines. In one elegant example, Turner and coworkers designed a biometallic whole cell catalyst composed of E coli BL21 cells overexpressing a monoamine oxidase (MAO-N-D5) with $\operatorname{Pd}(0)$ nanoparticles on the outer membrane. ${ }^{[12]}$ This biocomposite was used for the deracemization of 1-methyltetrahydroisoquinoline (MTQ) to $(R)$-MTQ through the imine intermediate with an $e e$ of up to $96 \%$, using $\mathrm{H}_{2}$ as electron donor for the reduction step in a sequential one-pot cyclic manner. This study illustrated the potential of combining the rapidly evolving synthetic biology techniques with novel nanometallic catalysts in a single organism for multistep transformations.

Although important developments have been made in the field, only recently new evolved enzymes have been found for sterically demanding molecules. Hence, the potential for using MAO-N variants was proved for the preparation of valuable biologically active molecules such as alkaloid crispine A. Initially, this approach with MAO-N-5 presented several limitations such as long reaction times (40 h) and low yields (48\%). In an attempt to improve this deracemization, several variants of MAO-N were prepared. ${ }^{[13]}$ After modelling crispine into the active site of MAO-N-5, four residues were highlighted as hot spots. Thus, the synthesis of $(R)$-crispine A was achieved in $2 \mathrm{~h}$ with an $e e$ of $>97 \%$ by using the MAO-N-9C variant, whose specific activity was 990-fold higher than MAO-N-5, together with the ammonia-borane complex as reducing agent in phosphate buffer at $37^{\circ} \mathrm{C}$. In another example, the asymmetric synthesis of several bioactive natural products such as Eleagnine, an alkaloid isolated from Chrysophyllum albidum and Leptaflorin, a psychedelic agent, was successfully performed using MAO-ND9. ${ }^{[114]}$ Furthermore, the development of a new D11 variant, which possessed high activity and excellent stereoselectivity toward bulky substrates, was accomplished. This enzyme was applied to the deracemization of Solefenacin, a competitive muscarinic acetylcholine receptor antagonist, and several Levocetirizine precursors, an antihistamine drug, thus expanding the substrate scope of MAO-N. For instance, the deracemization of 4-chlorobenzhydrylamine for the production of the $(R)$-configured amine was carried out with excellent enantiomeric excess (97\%) and moderate isolated yield (45\%, Scheme 18a). Also, the deracemization of several $\beta$-carbolines was achieved with MAO-N-D10 or MAO-N-D11, observing a strong influence on the enzyme stereoselectivity depending on the substitution pattern at position 1 (Scheme 18b). ${ }^{[115]}$ 
a)<smiles>CO[Nb](O)(O)c1ccc([C@H](N)c2ccc(Cl)cc2)cc1</smiles>

b)

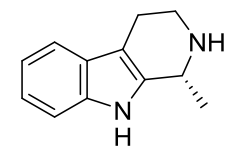

MAO-N-D10, 96\% $(R)$

MAO-N-D11, 99\% $(R)$<smiles>CCC1NCCc2c1[nH]c1ccccc21</smiles>

MAO-N-D10, 49\% (S)

MAO-N-D11, $12 \%(R)$

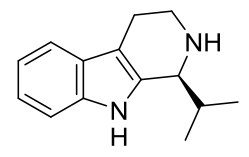

MAO-N-D10, 94\% (S)

MAO-N-D11, 22\% (S)

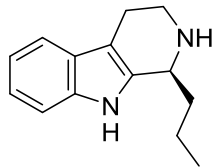

MAO-N-D10, 99\% (S)

MAO-N-D11, >99\% (S)

Scheme 18. a) Deracemization of a Levocitirizine motif using a MAO variant and the ammonia-borane complex. ${ }^{[14]}$ b) Deracemization of $\beta$-carbolines using MAO-N-D10 and MAO-N-D11. ${ }^{[15]}$

\section{Using new strategies}

Novel methods for the transformation of a racemic amine into an enantiopure product have been developed in the last few years by combining different (bio)catalysts in more complex systems. Inspired by synthetic and concurrent cascades, the cyclic deracemization of different amines was successfully achieved using a metalloenzyme, based on the supramolecular entrapment of an iridium complex within streptavidin acting as an artificial transfer hydrogenase (ATHase), together with a monoamino oxidase and a catalase (to quench the poisoning $\mathrm{H}_{2} \mathrm{O}_{2}$ ). A formate salt was added for regeneration. ${ }^{[16]}$ Thus, cyclic deracemization of secondary amines such as 1-methyltetrahydroisoquinoline or 2-cyclohexylpyrrolidine into the corresponding $(R)$-amines was investigated, achieving excellent conversions (99\%) and $e e(99 \%)$. In light of these results, authors demonstrated that these three enzymes could work cooperatively to prepare enantiopure amines in an orthogonal redox cascade. This approach holds promise for future applications in biosynthetic cascades.

In a recent example described by Turner and Kroutil groups, the deracemization of various benzylisoquinolines into the $(S)$-enantiomers, combining MAO-N D11 variant and the ammonia-borane complex, was performed in aqueous medium at $37^{\circ} \mathrm{C} .{ }^{[117]}$ As a further extension of this methodology, this biocatalyst was combined with a reductant agent (morpholine $\mathrm{BH}_{3}$ complex) and the berberine bridge enzyme (BBE) to yield $(S)$-berbines. ${ }^{[18]} \mathrm{BBE}$ is a selective oxidase which catalyzes the intramolecular C$\mathrm{C}$ coupling of $(S)$-reticuline leading to $(S)$-scoulerine. Only recently the substrate scope of BBE was studied, being suitable for the KR of these types of substrates. ${ }^{[119]}$ The deracemization of a series of benzylisoquinolines was achieved by simultaneous cyclic deracemization using the MAO/borane system coupled to a kinetic oxidative resolution catalyzed by BBE (Scheme 19). This three-step approach led to conversions up to $97 \%$ and excellent enantiopurities (ee >97\%) into the $(S)$-berbines. Moreover, the applicability of this system was demonstrated at preparative scale (150-165 mg) with similar results. This 
approach shows that challenging synthetic routes can be reconsidered into other more efficient and sustainable.<smiles>[R]c1cc2c(cc1[R])[C@@H](Cc1ccc([R])c(O)c1)N(C)CC2</smiles>
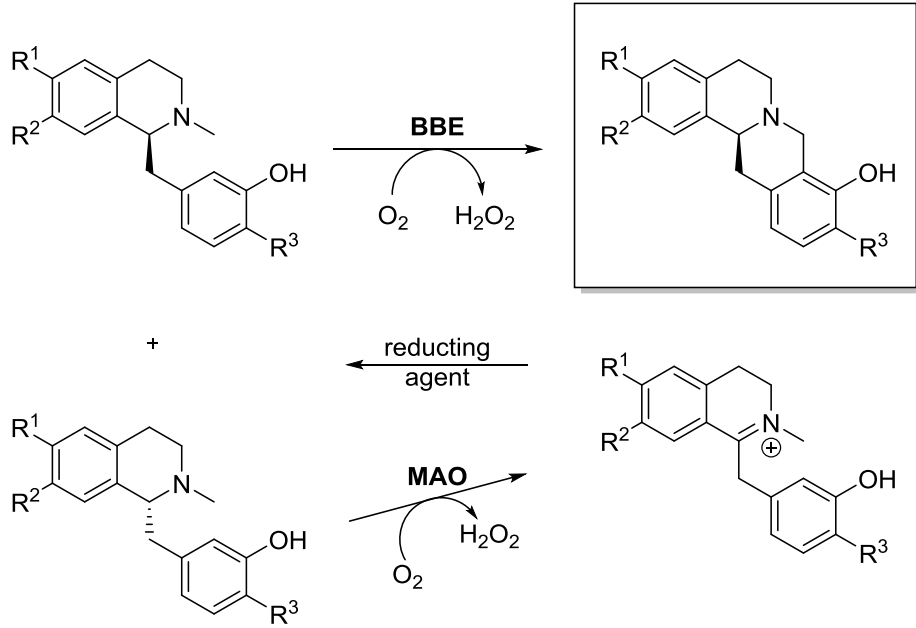

Scheme 19. Deracemization of a series of benzylisoquinolines into the corresponding $(S)$-berbines using

$$
\text { MAO and BBE. }{ }^{[118]}
$$

\section{Using an amino acid oxidase and a non-selective chemical reducing agent}

Patel's group also reported a cyclic deracemization route for the preparation of the previously mentioned amino acid 14. ${ }^{[105]}$ In this case, $(R)$-amino acid oxidase from Trigonopsis variabilis immobilized on Celite was combined with a chemical reductant (ammonia-borane complex) for the reduction of the imine intermediate. This cyclic deracemization was possible as the reduction of the imine intermediate was quicker than its hydrolysis. The final $(S)$-amino acid was obtained with a yield of $79 \%$ and $>99 \%$ ee.

Nowadays, there is a need for novel biocatalysts to cover new reactions or improve existing activities. In this sense, enzyme evolution to create new catalysts with desired functions is becoming an important tool in the last years. For example, an $(R)$-amino oxidase has recently been redesigned starting from porcine kidney D-amino acid oxidase (pkDAO). ${ }^{[120]}$ The engineered enzyme obtained by directed evolution, showed a high $R$-specificity towards benzylic substrates and was further applied to the deracemization of $\alpha$-methylbenzylamine in the presence of $\mathrm{NaBH}_{4}$, transformation that had not been previously described for amino acid oxidases. This work opens the door for future applications in the synthesis of chiral and more complex amines via deracemization.

\subsection{Cyclic deracemizations over sulfoxide derivatives using oxidoreductases}

Parvulescu and co-workers reported the deracemization of sulfoxides by combining E. coli cells, which performed the selective reduction of these compounds into the sulfides, followed by a non-selective oxidation with a heterogeneous catalyst $\left(\mathrm{Ta}_{2} \mathrm{O}_{5}-\mathrm{SiO}_{2}\right) \cdot{ }^{[121]}$ In this case, due to incompatibility of both reactions, they were performed in a sequential manner. For instance, after three cycles, $(S)$-methyl $p$-tolyl sulfoxide was prepared in 56\% yield and $98 \%$ ee (Scheme 20). 


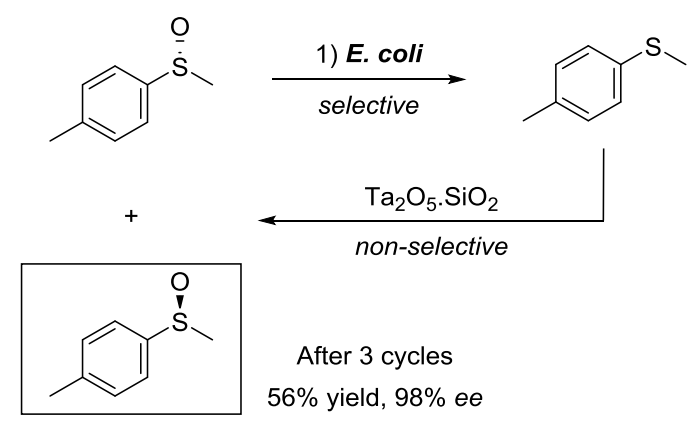

Scheme 20. Sequential deracemization of methyl $p$-tolyl sulfoxide via enzymatic reduction plus metalcatalyzed oxidation. ${ }^{[121]}$

\section{Enantioconvergent processes}

These strategies involve transformations in which both enantiomers of a racemic mixture are converted into the same enantiomer of a product or a homochiral mixture of products through different reaction pathways, one via retention of configuration and the second one through inversion of configuration (Scheme 1). ${ }^{[122]}$ Therefore for this strategy an inverting enzyme can be used. There are some biocatalysts capable to perform this transformation: glycosidases, haloalkane dehalogenases, epoxide hydrolases and alkylsulfatases.

\section{Using haloalkane dehalogenases}

Janssen and Feringa groups described a chemoenzymatic enantioconvergent process for the synthesis of enantiopure $\alpha$-substituted amides using haloalkane dehalogenases. ${ }^{[123]}$ In this study, $\alpha$-bromoamides reacted with high enantioselectivity in the presence of haloalkane dehalogenase from Bradyrhizobium japonicum USDA110 (DbjA), affording a homochiral mixture consisting of the remaining $(S)$-brominated substrate enantiomer and the $(S)$-alcohol product coming from the inverting reaction over the $(R)$ enantiomer (Scheme 21). Then, after mesylation, the $\alpha$-bromide and the $\alpha$-mesyloxy amides were finally treated with several nucleophiles in a $\mathrm{S}_{\mathrm{N}} 2$ process to achieve the substituted $(R)$-amides. These products were obtained in high yields (up to $96 \%$ after the three steps) and excellent $e e$ (up to $98 \%$ ).

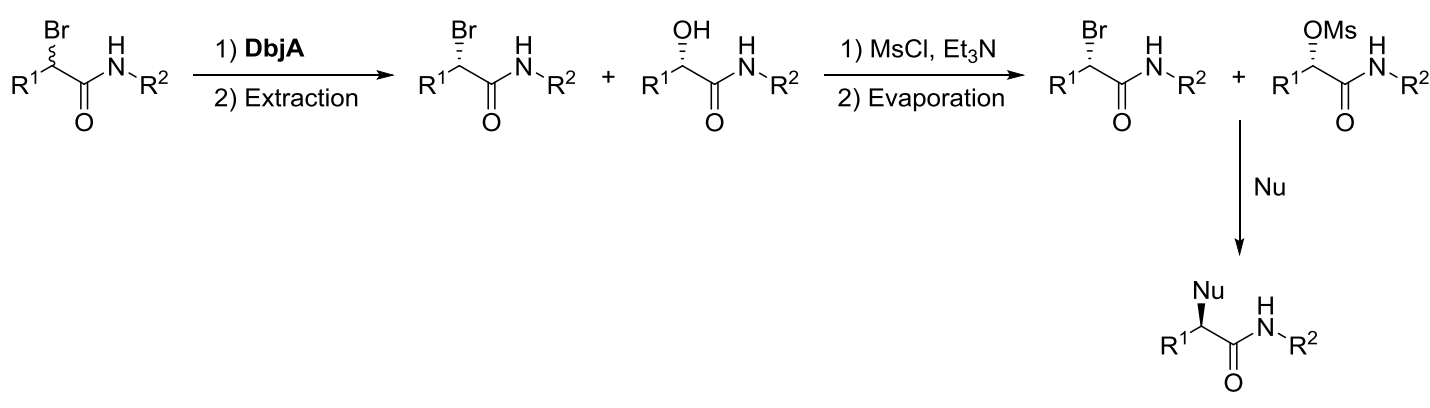

$\mathrm{Nu}=\mathrm{N}_{3}, \mathrm{NHBn}, \mathrm{OPh}, \mathrm{SEt}$

Scheme 21. Enantioconvergent strategy for the preparation of enantiopure $\alpha$-substituted amides using haloalkane dehalogenases in the inverting step. ${ }^{[123]}$ 


\section{Using alkylsulfatases}

Other stereoselective inverting enzymes are alkylsulfatases, which have been identified as member of the $\beta$-metallolactamase family and that catalyze the nucleophilic displacement of $\mathrm{HSO}_{4}^{-}$by water via $\mathrm{OH}^{-[124]}$ Among them, Pseudomonas sp. DSM 6611 alkylsulfatase (Pisa1) has widely been used in the deracemization of sec-alcohols. In an interesting report from Faber's group, this enzyme was overexpressed in E. coli and used for the preparation of alkyl-alkyl (S)-configured sec-alcohols together with the non-reacting $(S)$-sulfate esters. The latter can be then transformed into the $(S)$-alcohols by chemical deprotection under acidic conditions. ${ }^{[125]}$ In a later study, the same authors investigated the scope of this enzyme finding that it showed good stereoselectivity in the hydrolysis of a variety of sulfate esters such as aromatic, olefinic and acetylenic sulfates, especially when bearing two groups of different size. Moreover, the addition of DMSO (20\% v/v) proved to be very convenient in order to obtain better enantioselectivities. ${ }^{[126]}$ This enzyme was also employed to successfully deracemize hex-5-en-2-ol into the $(S)$-enantiomer, precursor of a lasiodiplodin derivative, which displays antileukemia activity. ${ }^{[127]}$

Recently, an elegant report for the one-pot deracemization of various sec-alcohols was described in the presence of two stereocomplementary sulfatases (Scheme 22). ${ }^{[128]}$ This study was possible due to the combination of the retaining and inverting features of two sulfatases by sequential or simultaneous enantioconvergent hydrolysis of the corresponding sulfate esters. The key of this approach was the stereochemical properties of arylsulfatase from Pseudomonas aeruginosa (PAS), an $R$-selective enzyme working with retention of configuration, and the $S$-preference showed by Pisal with inversion of configuration. Using this protocol, the deracemization of sulfate esters to form the corresponding unprotected $(R)$-alcohols could be achieved with conversions higher than $80 \%$ and $e e$ higher than $90 \%$.

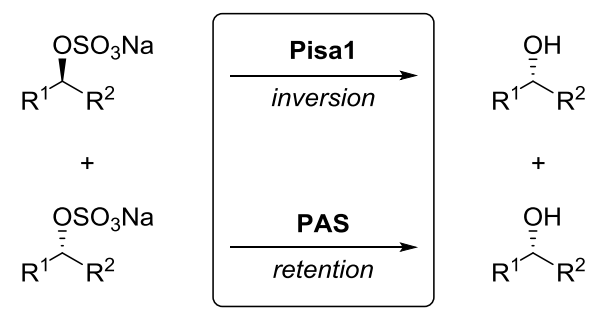

Scheme 22. One-pot deracemization of sec-alcohols using simultaneously two stereocomplementary

$$
\text { alkylsulfatases. }{ }^{[128]}
$$

\section{Using epoxide hydrolases}

This class of enzymes has been used in enantioconvergent processes for the preparation of enantiopure diols in a theoretical yield of $100 \%$ starting from the corresponding racemic epoxides. This is possible if one of the enantiomers reacts with retention of configuration, while the other one is hydrolyzed with inversion. Kotik et al. evolved the epoxide hydrolase (EH) from Aspergillus niger M200 by saturation mutagenesis. ${ }^{[129]}$ This was performed at nine sites close to the binding pocket and the substrate access tunnel. The final EH mutant was capable to transform racemic styrene oxide and $p$-chlorostyrene oxide to the corresponding $(R)$-diols with an $e e$ of $70 \%$ for both substrates. In an attempt to enhance the products $e e$, a sequential bi-enzymatic reaction was performed combining the wild-type enzyme and several 
evolved mutants, observing a significant increase of the $e e$ values from $70 \%$ to $88-91 \%$. In a subsequent work, Kau2 EH retrieved from the genomic DNA of a microbial community, was also mutated for the selective enantioconvergent hydrolysis of $p$-chlorostyrene oxide into the $(R)$-diol, finding a variant that improved the selectivity of this transformation up to $93 \% e e .^{[130]}$ The preparation of these compounds (Scheme 23) was also achieved by a sequential reaction using recombinant $E$. coli cells overexpressing two regio- and enantiocomplementary EHs, namely Caulobacter crescentus $(\mathrm{CcEH})$ and Mugil cephalus $(\mathrm{McEH})$, obtaining $(R)-1$-phenyl-1,2-ethanediol in $95 \%$ yield and $90 \% e e .^{[131]}$

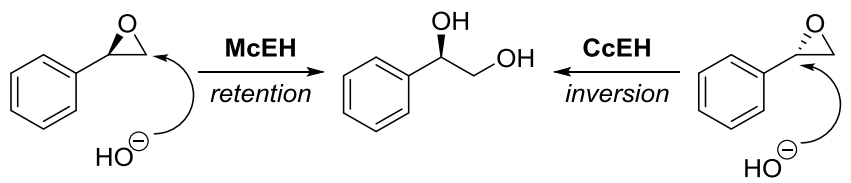

Scheme 23. Strategy for the preparation of enantiopure $(R)-1,2$-phenylethanediol using two enantiocomplementary epoxide hydrolases. ${ }^{[131]}$

\section{Using lipases}

The stereoconvergence of a racemate into a single enantiopure product can also be achieved through a sequential transformation performing a lipase-catalyzed resolution followed by a chemical inversion of the remaining or formed alcohol by, i.e. a Mitsunobu reaction. Matsumoto and co-workers reported the deracemization of acetylated 1,2-diols $O$-tosylated at $\beta$-position combining a lipase-catalyzed hydrolysis and a Mitsunobu reaction. ${ }^{[132]}$ The authors examined the hydrolysis of these bulky substrates catalyzed by PS lipase, and made use of immobilized triphenylphospine in polystyrene for the Mitsunobu inversion. Using this protocol, $(S)$-2-acetoxy-6-benzyloxytosylate 15, key core of alkaloid $(S)$-coniine, was synthesized in very good overall yield (82\%) and 98\% ee (Scheme 24). In another example, secondary benzylic acetates were deracemized employing a two-step protocol consisting of a CAL-B-catalyzed hydrolysis followed by a Mitsunobu reaction obtaining the $(S)$-acetates in high yields (70-89\%) and moderate to excellent $e e(57-99 \%) .{ }^{[133]}$

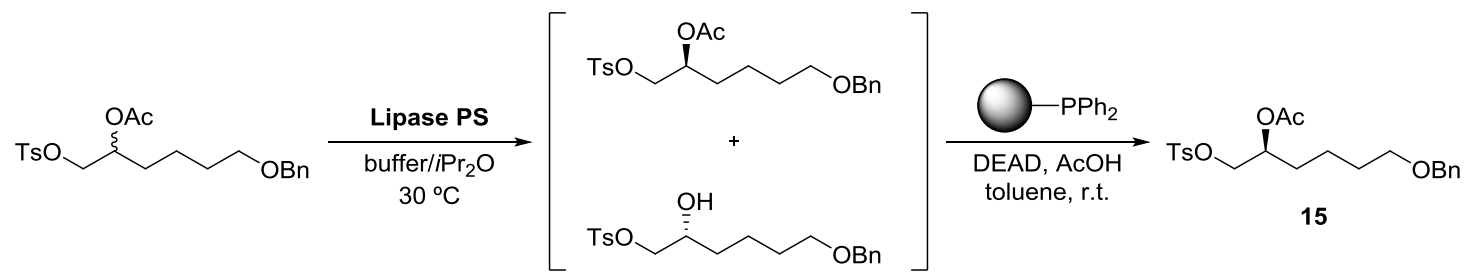

Scheme 24. Preparation of $(S)$-15, key intermediate of $(S)$-coniine, via lipase-catalyzed hydrolysis plus Mitsunobu inverting protocol. ${ }^{[132]}$ 


\section{Summary and Outlook}

Since 80 s biocatalyzed kinetic resolutions of racemates have been systematically studied to obtain compounds in enantiopure form. This is a well-established methodology as a great number of biocatalysts are easily available. Unfortunately, in these processes $50 \%$ of the starting material must be discarded unless the opposite antipode is also of interest. Therefore, in order to complete the job, yields close to $100 \%$ are highly desired to obtain optically pure products. To achieve this purpose, different techniques have been developed to overcome the yield limitation imposed by KRs. Among the methods that are already implemented at both academic and industrial level, dynamic kinetic resolutions, deracemizations via stereoinversion, cyclic deracemizations and enantioconvergent processes can be mentioned. DKRs are well-implemented protocols and those operating with a metal-catalyst as racemizing agent, are the most used to deracemize alcohols or amines coupled to a hydrolase. While for the first ones Ru catalysts have been the most commonly employed, for amino compounds palladium-based systems are usually exploited. In the case of DKRs over carboxylic or carbonylic derivatives, due to the high acidity displayed by the proton at $\alpha$-position, a good choice is the application of basic conditions to racemize these compounds. Nowadays the modification of enzymes through molecular engineering and the application of very promising biocatalysts such transaminases are providing novel efficient protocols. Also, the design of new metal catalysts or metal-enzyme composites is allowing the access to highly valuable compounds.

In the case of stereoinversions, these systems are more usual when working with oxidoreductases to deracemize alcohols or amines, but recently transaminases have also been utilized. In this case whole cell systems or two stereoselective and enantiocomplementary biocatalysts must be selected. If one of the processes is not selective, still deracemization can be attained in a cyclic manner. Good examples of this methodology are amino oxidases coupled to a non-selective chemical reagent, but also very recently the employment of metalloenzymes with hydrogen transfer activity has been envisaged as a potential alternative to these protocols. Enantioconvergent systems are probably the less developed deracemization techniques but still they can find interesting applications when using inverting enzymes such dehalogenases, epoxide hydrolases and alkylsulfatases in order to obtain, e.g. chiral alcohol or diol derivatives.

As a result of the tremendous advances in the biocatalysis field especially due to protein engineering, DNA sequencing and computational design, impressive novel capabilities can be conferred to enzymes. Obviously, these redesigned biocatalysts can be successfully implemented in deracemization protocols to access challenging targets, e.g. sterically demanding, in excellent yields and enantioselectivities. Moreover, the need for more sustainable processes based on highly efficient transformations, is turning the attention into the development of systems where the combination of several (bio)catalytic steps can be performed in one-pot, designing multi-step catalytic cascade or tandem processes avoiding the isolation of the corresponding intermediates. Accordingly, the understanding of enzymatic mechanisms, improvements in the enzyme stability, and the combination of several (bio)catalysts in one-pot or stepwise fashion is allowing in many cases a dramatic progress in chemical synthesis and will help biocatalysts to spread into industry. 


\section{Acknowledgments}

I.L. thanks the Spanish Ministerio de Ciencia e Innovación (MICINN) for personal funding (Ramón y Cajal Program). Financial support of this work by the Spanish MICINN (Projects CTQ2011-24237 and CTQ2013-44153) is gratefully acknowledged.

\section{Conflict of Interest}

Authors declare no conflict of interest.

\section{References}

[1] Anastas, P.T.; Warner, J.C. Green chemistry: Theory and practice; Oxford University Press: Oxford, 1998.

[2] Bornscheuer, U.T.; Huisman, G.W.; Kazlauskas, R.J.; Lutz, S.; Moore, J.C.; Robins, K. Engineering the third wave of biocatalysis. Nature, 2012, 485, 185-194.

[3] García-Urdiales, E.; Alfonso, I.; Gotor, V. Update 1 of: Enantioselective enzymatic desymmetrizations in organic synthesis. Chem. Rev., 2011, 111, PR110-PR180.

[4] Stecher, H.; Faber, K. Biocatalytic deracemization techniques. Dynamic resolutions and stereoinversions. Synthesis, 1997, 1-16.

[5] Matsushita, H.; Noguchi, M.; Saburi, M.; Yoshikawa, S. Optical activation of $\alpha$-substituted carbonyl compounds by asymmetric transformation. Bull. Chem. Soc. Jpn., 1975, 48, 3715-3717.

[6] Deol, B.S.; Ridley, D.D.; Simpson, G.W. Asymmetric reduction of carbonyl compounds by yeast. II. Preparation of optically active $\alpha$ - and $\beta$-hydroxy carboxylic acid derivatives. Aust. J. Chem., 1976, 29, 2459-2467.

[7] Gadler, P.; Glueck, S.M.; Kroutil, W.; Nestl, B.M.; Larissegger-Schnell, B.; Ueberbacher, B.T.; Wallner, S.R.; Faber, K. Biocatalytic approaches for the quantitative production of single stereoisomers from racemates. Biochem. Soc. Trans., 2006, 34, 296-300.

[8] Simeó, Y.; Kroutil, W.; Faber, K. In: Biocatalysis in the pharmaceutical and biotechnology industries; Patel, R.N., Ed.; CRC Press: Boca Raton, 2007, pp. 27-51.

[9] Martin-Matute, B.; Baeckvall, J.-E. In: Organic synthesis with enzymes in non-aqueous media; Carrea, G.; Riva, S., Eds.; Wiley-VCH: Weinheim, 2008, pp. 113-144.

[10] Turner, N.J. In: Asymmetric organic synthesis with enzymes; Gotor, V.; Alfonso, I.; García-Urdiales, E., Eds.; Wiley-VCH: Weinheim, 2008, pp. 115-131.

[11] Tessaro, D.; Molla, G.; Pollegioni, L.; Servi, S. In: Modern biocatalysis; Fessner, W.-D.; Anthonsen, T., Eds.; Wiley-VCH: Weinheim, 2009, pp. 195-228.

[12] Turner, N.J. Deracemization methods. Curr. Opin. Chem. Biol., 2010, 14, 115-121.

[13] Rachwalski, M.; Vermue, N.; Rutjes, F.P.J.T. Recent advances in enzymatic and chemical deracemisation of racemic compounds. Chem. Soc. Rev., 2013, 42, 9268-9282. 
[14] Noyori, R.; Ikeda, T.; Ohkuma, T.; Widhalm, M.; Kitamura, M.; Takaya, H.; Akutagawa, S.; Sayo, N.; Saito, T.; Taketomi, T.; Kumobayashi, H. Stereoselective hydrogenation via dynamic kinetic resolution. J. Am. Chem. Soc., 1989, 111, 9134-9135.

[15] Lee, J.H.; Han, K.; Kim, M.-J.; Park, J. Chemoenzymatic dynamic kinetic resolution of alcohols and amines. Eur. J. Org. Chem., 2010, 999-1015.

[16] Pellissier, H. Recent developments in dynamic kinetic resolution. Tetrahedron, 2011, 67, 3769-3802.

[17] Kim, Y.-W.; Park, J.-W.; Kim, M.-J. Dynamic kinetic resolution of amines and amino acids by enzyme-metal cocatalysis. ChemCatChem, 2011, 3, 271-277.

[18] Hussain, I.; Bäckvall, J.E. In: Enzyme catalysis in organic synthesis; Drauz, K.; Gröger, H.; May, O., Eds.; Wiley-VCH: Weinheim, 3rd ed., 2012, pp. 1777-1806.

[19] Rebolledo, F.; González-Sabín, J.; Gotor, V. In: Stereoselective synthesis of drugs and natural products; Andrushko, V.; Andrushko, N., Eds.; John Wiley \& Sons: Hoboken, 2013, pp. 1683-1711.

[20] Tan, D.S.; Günter, M.M.; Drueckhammer, D.G. Enzymatic resolution coupled with substrate racemization using a thioester substrate. J. Am. Chem. Soc., 1995, 117, 9093-9094.

[21] Hoyos, P.; Pace, V.; Alcántara, A.R. Dynamic kinetic resolution via hydrolase-metal combo catalysis in stereoselective synthesis of bioactive compounds. Adv. Synth. Catal., 2012, 354, 2585-2611.

[22] Larsson, A.L.E.; Persson, B.A.; Bäckvall, J.-E. Enzymatic resolution of alcohols coupled with ruthenium-catalyzed racemization of the substrate alcohol. Angew. Chem. Int. Ed. Engl., 1997, 36, 12111212.

[23] Menasche, N.; Shvo, Y. Catalytic disproportionation of aldehydes with ruthenium complexes. Organometallics, 1991, 10, 3885-3891.

[24] Hoyos, P.; Pace, V.; Sinisterra, J.V.; Alcántara, A.R. Chemoenzymatic synthesis of chiral unsymmetrical benzoin esters. Tetrahedron, 2011, 67, 7321-7329.

[25] Hoyos, P.; Quezada, M.A.; Sinisterra, J.V.; Alcántara, A.R. Optimised dynamic kinetic resolution of benzoin by a chemoenzymatic approach in 2-MeTHF. J. Mol. Catal. B: Enzym., 2011, 72, $20-24$.

[26] Lihammar, R.; Millet, R.; Bäckvall, J.-E. Enzyme- and ruthenium-catalyzed dynamic kinetic resolution of functionalized cyclic allylic alcohols. J. Org. Chem., 2013, 78, 12114-12120.

[27] Martín-Matute, B.; Edin, M.; Bogár, K.; Bäckvall, J.-E. Highly compatible metal and enzyme catalysts for efficient dynamic kinetic resolution of alcohols at ambient temperature. Angew. Chem. Int. Ed., 2004, 43, 6535-6539.

[28] Träff, A.; Lihammar, R.; Bäckvall, J.-E. A chemoenzymatic dynamic kinetic resolution approach to enantiomerically pure ( $R$ )- and (S)-duloxetine. J. Org. Chem., 2011, 76, 3917-3921.

[29] Lihammar, R.; Millet, R.; Bäckvall, J.-E. An efficient dynamic kinetic resolution of N-heterocyclic 1,2-amino alcohols. Adv. Synth. Catal., 2011, 353, 2321-2327.

[30] Warner, M.C.; Nagendiran, A.; Bogár, K.; Bäckvall, J.-E. Enantioselective route to ketones and lactones from exocyclic allylic alcohols via metal and enzyme catalysis. Org. Lett., 2012, 14, 5094-5097. [31] Warner, M.C.; Shevchenko, G.A.; Jouda, S.; Bogár, K.; Bäckvall, J.-E. Dynamic kinetic resolution of homoallylic alcohols: Application to the synthesis of enantiomerically pure 5,6-dihydropyran-2-ones and $\delta$-lactones. Chem. Eur. J., 2013, 19, 13859-13864. 
[32] Shuklov, I.A.; Dubrovina, N.V.; Schulze, J.; Tietz, W.; Börner, A. Synthesis of (R)-propane-1,2-diol from lactides by dynamic kinetic resolution. Tetrahedron Lett., 2014, 55, 3495-3497.

[33] Päiviö, M.; Mavrynsky, D.; Leino, R.; Kanerva, L.T. Dynamic kinetic resolution of a wide range of secondary alcohols: Cooperation of dicarbonylchlorido(pentabenzylcyclopentadienyl)ruthenium and CAL-B. Eur. J. Org. Chem., 2011, 1452-1457.

[34] Das, T.; Nanda, S. Chemoenzymatic total synthesis of stagonolide-E. Tetrahedron Lett., 2012, 53, 256-258

[35] Rej, R.K.; Jana, A.; Nanda, S. Asymmetric synthesis of naturally occurring nonenolide xyolide through cross metathesis and macrolactonization reaction. Tetrahedron, 2014, 70, 2634-2642.

[36] Kim, S.; Choi, Y.K.; Hong, J.; Park, J.; Kim, M.-J. Candida antarctica lipase A and Pseudomonas stutzeri lipase as a pair of stereocomplementary enzymes for the resolution of 1,2-diarylethanols and 1,2diarylethanamines. Tetrahedron Lett., 2013, 54, 1185-1188.

[37] Merabet-Khelassi, M.; Vriamont, N.; Aribi-Zouioueche, L.; Riant, O. Racemization of secondary alcohols catalyzed by ruthenium: Application to chemoenzymatic dynamic resolution. Tetrahedron: Asymmetry, 2011, 22, 1790-1796.

[38] Agrawal, S.; Martínez-Castro, E.; Marcos, R.; Martín-Matute, B. Readily available ruthenium complex for efficient dynamic kinetic resolution of aromatic $\alpha$-hydroxy ketones. Org. Lett., 2014, 16, 2256-2259.

[39] Engström, K.; Vallin, M.; Syrén, P.-O.; Hult, K.; Bäckvall, J.-E. Mutated variant of Candida antarctica lipase B in $(S)$-selective dynamic kinetic resolution of secondary alcohols. Org. Biomol. Chem., 2011, 9, 81-82.

[40] Ema, T.; Nakano, Y.; Yoshida, D.; Kamata, S.; Sakai, T. Redesign of enzyme for improving catalytic activity and enantioselectivity toward poor substrates: Manipulation of the transition state. Org. Biomol. Chem., 2012, 10, 6299-6308.

[41] Kim, H.; Choi, Y.K.; Lee, J.; Lee, E.; Park, J.; Kim, M.-J. Ionic-surfactant-coated Burkholderia cepacia lipase as a highly active and enantioselective catalyst for the dynamic kinetic resolution of secondary alcohols. Angew. Chem. Int. Ed., 2011, 50, 10944-10948.

[42] Kim, C.; Lee, J.; Cho, J.; Oh, Y.; Choi, Y.K.; Choi, E.; Park, J.; Kim, M.-J. Kinetic and dynamic kinetic resolution of secondary alcohols with ionic-surfactant-coated Burkholderia cepacia lipase: Substrate scope and enantioselectivity. J. Org. Chem., 2013, 78, 2571-2578.

[43] Sato, Y.; Kayaki, Y.; Ikariya, T. Efficient dynamic kinetic resolution of racemic secondary alcohols by a chemoenzymatic system using bifunctional iridium complexes with $\mathrm{C}-\mathrm{N}$ chelate amido ligands. Chem. Commun., 2012, 48, 3635-3637.

[44] Egi, M.; Sugiyama, K.; Saneto, M.; Hanada, R.; Kato, K.; Akai, S. A mesoporous-silica-immobilized oxovanadium cocatalyst for the lipase-catalyzed dynamic kinetic resolution of racemic alcohols. Angew. Chem. Int. Ed., 2013, 52, 3654-3658.

[45] Li, X.; Shi, Y.; Wang, Z.; Zhang, Y.; Tang, Y. Catalytic performance of H- $\beta$ nanozeolite microspheres in one-pot dynamic kinetic resolution of aromatic sec-alcohols. J. Catal., 2012, 288, 24-32. 
[46] Izquierdo, D.F.; Bernal, J.M.; Burguete, M.I.; García-Verdugo, E.; Lozano, P.; Luis, S.V. An efficient microwave-assisted enzymatic resolution of alcohols using a lipase immobilised on supported ionic liquid-like phases (SILLPs). RSC Adv., 2013, 3, 13123-13126.

[47] Xu, G.; Wang, L.; Chen, Y.; Cheng, Y.; Wu, J.; Yang, L. Highly efficient dynamic kinetic resolution of secondary aromatic alcohols using a low-cost solid super acid as a racemization catalyst. Tetrahedron Lett., 2013, 54, 5026-5030.

[48] Wang, J.; Do, D.-M.; Chuah, G.-K.; Jaenicke, S. Core-shell composite as the racemization catalyst in the dynamic kinetic resolution of secondary alcohols. ChemCatChem, 2013, 5, 247-254.

[49] Nemoto, H.; Tanimoto, K.; Kanao, Y.; Omura, S.; Kita, Y.; Akai, S. Protecting-group-free catalytic asymmetric total synthesis of (-)-rosmarinecine. Tetrahedron, 2012, 68, 7295-7301.

[50] Dynamic Combinatorial Chemistry; Reek, J.; Otto, S., Eds.; Wiley-VCH: Weinheim, 2010.

[51] Sakulsombat, M.; Vongvilai, P.; Ramström, O. In situ evaluation of lipase performances through dynamic asymmetric cyanohydrin resolution. Org. Biomol. Chem., 2011, 9, 1112-1117.

[52] Sakulsombat, M.; Zhang, Y.; Ramström, O. Dynamic asymmetric hemithioacetal transformation by lipase-catalyzed $\gamma$-lactonization: In situ tandem formation of 1,3-oxathiolan-5-one derivatives. Chem. Eur. J., 2012, 18, 6129-6132.

[53] Zhang, Y.; Hu, L.; Ramström, O. Double parallel dynamic resolution through lipase-catalyzed asymmetric transformation. Chem. Commun., 2013, 49, 1805-1807.

[54] Hu, L.; Schaufelberger, F.; Zhang, Y.; Ramström, O. Efficient asymmetric synthesis of lamivudine via enzymatic dynamic kinetic resolution. Chem. Commun., 2013, 49, 10376-10378.

[55] Zhang, Y.; Schaufelberger, F.; Sakulsombat, M.; Liu, C.; Ramström, O. Asymmetric synthesis of 1,3-oxathiolan-5-one derivatives through dynamic covalent kinetic resolution. Tetrahedron, 2014, 70, 3826-3831.

[56] Reetz, M.T.; Schimossek, K. Lipase-catalyzed dynamic kinetic resolution of chiral amines. Use of palladium as the racemization catalyst. Chimia, 1996, 50, 668-669.

[57] Choi, E.; Kim, Y.; Ahn, Y.; Park, J.; Kim, M.-J. Highly enantioselective enzymatic resolution of aromatic $\beta$-amino acid amides with Pd-catalyzed racemization. Tetrahedron: Asymmetry, 2013, 24, 14491452.

[58] Qian, X.; Jiang, Z.; Lin, X.; Wu, Q. Tandem dynamic kinetic resolution and enzymatic polycondensation to synthesize mPEG-functionalized poly(amine-co-ester)-type chiral prodrugs. $J$. Polym. Sci. Pol. Chem., 2013, 51, 2049-2057.

[59] Cheng, G.; Xia, B.; Wu, Q.; Lin, X. Chemoenzymatic dynamic kinetic resolution of $\alpha$ trifluoromethylated amines: Influence of substitutions on the reversed stereoselectivity. RSC Adv., 2013, $3,9820-9828$.

[60] Xu, G.; Dai, X.; Fu, S.; Wu, J.; Yang, L. Efficient dynamic kinetic resolution of arylamines with Pd/layered double-hydroxide-dodecyl sulfate anion for racemization. Tetrahedron Lett., 2014, 55, 397402.

[61] Engström, K.; Johnston, E.V.; Verho, O.; Gustafson, K.P.J.; Shakeri, M.; Tai, C.-W.; Bäckvall, J.-E. Co-immobilization of an enzyme and a metal into the compartments of mesoporous silica for cooperative tandem catalysis: An artificial metalloenzyme. Angew. Chem. Int. Ed., 2013, 52, 14006-14010. 
[62] Gustafson, K.P.J.; Lihammar, R.; Verho, O.; Engström, K.; Bäckvall, J.-E. Chemoenzymatic dynamic kinetic resolution of primary amines using a recyclable palladium nanoparticle catalyst together with lipases. J. Org. Chem., 2014, 79, 3747-3751.

[63] Rodríguez-Mata, M.; Gotor-Fernández, V.; González-Sabín, J.; Rebolledo, F.; Gotor, V. Straightforward preparation of biologically active 1-aryl- and 1-heteroarylpropan-2-amines in enantioenriched form. Org. Biomol. Chem., 2011, 9, 2274-2278.

[64] Morán-Ramallal, R.; Gotor-Fernández, V.; Laborda, P.; Sayago, F.J.; Cativiela, C.; Gotor, V. Dynamic kinetic resolution of 1,3-dihydro- $2 H$-isoindole-1-carboxylic acid methyl ester: Asymmetric transformations toward isoindoline carbamates. Org. Lett., 2012, 14, 1696-1699.

[65] Hietanen, A.; Lundell, K.; Kanerva, L.T.; Liljeblad, A. Advances in the kinetic and dynamic kinetic resolution of piperazine-2-carboxylic acid derivatives with Candida antarctica lipase A; structural requirements for enantioselective N-acylation. Arkivoc, 2012, 60-74.

[66] Zhang, Y.; Ramström. O. Thiazolidinones derived from dynamic systemic resolution of complex reversible-reaction networks. Chem. Eur. J., 2014, 20, 3288-3291.

[67] Berkessel, A.; Jurkiewicz, I.; Mohan, R. Enzymatic dynamic kinetic resolution of oxazinones: A new approach to enantiopure $\beta^{2}$-amino acids. ChemCatChem, 2011, 3, 319-330.

[68] Truppo, M.D.; Hughes, G. Development of an improved immobilized CAL-B for the enzymatic resolution of a key intermediate to odanacatib. Org. Process Res. Dev., 2011, 15, 1033-1035.

[69] Chavez-Flores, D.; Salvador, J.M. Facile conversion of racemic ibuprofen to $(S)$-ibuprofen. Tetrahedron: Asymmetry, 2012, 23, 237-239.

[70] Bongen, P.; Pietruszka, J.; Simon, R.C. Dynamic kinetic resolution of 2,3-dihydrobenzo[b]furans: Chemoenzymatic synthesis of analgesic agent BRL 37959. Chem. Eur. J., 2012, 18, 11063-11070.

[71] Wiggins, K.M.; Bielawski, C.W. A mechanochemical approach to deracemization. Angew. Chem. Int. Ed., 2012, 51, 1640-1643.

[72] Chaplin, D.A.; Fox, M.E.; Kroll, S.H.B. Dynamic kinetic resolution of dehydrocoronamic acid. Chem. Commun., 2014, 50, 5858-5860.

[73] D’Arrigo, P.; Cerioli, L.; Servi, S.; Viani, F.; Tessaro, D. Synergy between catalysts: enzymes and bases. DKR of non-natural amino acids derivatives. Catal. Sci. Technol., 2012, 2, 1606-1616.

[74] D’Arrigo, P.; Cerioli, L.; Fiorati, A.; Servi, S.; Viani, F.; Tessaro, D. Naphthyl-L- $\alpha$-amino acids via chemo-enzymatic dynamic kinetic resolution. Tetrahedron: Asymmetry, 2012, 23, 938-944.

[75] Baxter, S.; Royer, S.; Grogan, G.; Brown, F.; Holt-Tiffin, K.E.; Taylor, I.N.; Fotheringham, I.G.; Campopiano, D.J. An improved racemase/acylase biotransformation for the preparation of enantiomerically pure amino acids. J. Am. Chem. Soc., 2012, 134, 19310-19313.

[76] Qiu, J.; Su, E.; Wang, W.; Wei, D. High yield synthesis of D-phenylglycine and its derivatives by nitrilase mediated dynamic kinetic resolution in aqueous-1-octanol biphasic system. Tetrahedron Lett., 2014, 55, 1448-1451.

[77] Qiu, J.; Su, E.; Wang, W.; Wei, D. Efficient asymmetric synthesis of D- $N$-formyl-phenylglycine via cross-linked nitrilase aggregates catalyzed dynamic kinetic resolution. Catal. Commun., 2014, 51, 19-23. 
[78] Zhang, C.-S.; Zhang, Z.-J.; Li, C.-X.; Yu, H.-L.; Zheng, G.-W.; Xu, J.-H. Efficient production of $(R)$ - $o$-chloromandelic acid by deracemization of $o$-chloromandelonitrile with a new nitrilase mined from Labrenzia aggregata. Appl. Microbiol. Biotechnol., 2012, 95, 91-99.

[79] Yasukawa, K.; Asano, Y. Enzymatic synthesis of chiral phenylalanine derivatives by a dynamic kinetic resolution of corresponding amide and nitrile substrates with a multi-enzyme system. Adv. Synth. Catal., 2012, 354, 3327-3332.

[80] Cuetos, A.; Rioz-Martínez, A.; Bisogno, F.R.; Grischek, B.; Lavandera, I.; de Gonzalo, G.; Kroutil, W.; Gotor, V. Access to enantiopure $\alpha$-alkyl- $\beta$-hydroxy esters through dynamic kinetic resolutions employing purified/overexpressed alcohol dehydrogenases. Adv. Synth. Catal., 2012, 354, 1743-1749.

[81] Xu, F.; Chung, J.Y.L.; Moore, J.C.; Liu, Z.; Yoshikawa, N.; Hoerrner, R.S.; Lee, J.; Royzen, M.; Cleator, E.; Gibson, A.G.; Dunn, R.; Maloney, K.M.; Alam, M.; Goodyear, A.; Lynch, J.; Yasuda, N.; Devine, P.N. Asymmetric synthesis of cis-2,5-disubstituted pyrrolidine, the core scaffold of $\beta_{3}$-AR agonists. Org. Lett., 2013, 15, 1342-1345.

[82] Kalaitzakis, D.; Smonou, I. Chemoenzymatic synthesis of stegobinone and stegobiol, components of the natural sex pheromone of the drugstore beetle (Stegobium paniceum L.). Eur. J. Org. Chem., 2012, 43-46.

[83] Mangas-Sánchez, J.; Busto, E.; Gotor, V.; Gotor-Fernández, V. One-pot synthesis of enantiopure 3,4-dihydroisocoumarins through dynamic reductive kinetic resolution processes. Org. Lett., 2013, 15, 3872-3875.

[84] Quaglia, D.; Pori, M.; Galletti, P.; Emer, E.; Paradisi, F.; Giacomini, D. His-tagged horse liver alcohol dehydrogenase: Immobilization and application in the bio-based enantioselective synthesis of $(S)$ arylpropanols. Process Biochem., 2013, 48, 810-818.

[85] Könst, P.; Merkens, H.; Kara, S.; Kochius, S.; Vogel, A.; Zuhse, R.; Holtmann, D.; Arends, I.W.C.E.; Hollmann, F. Enantioselective oxidation of aldehydes catalyzed by alcohol dehydrogenase. Angew. Chem. Int. Ed., 2012, 51, 9914-9917.

[86] Rioz-Martínez, A.; Cuetos, A.; Rodríguez, C.; de Gonzalo, G.; Lavandera, I.; Fraaije, M.W.; Gotor, V. Dynamic kinetic resolution of $\alpha$-substituted $\beta$-ketoesters catalyzed by Baeyer-Villiger monooxygenases: Access to enantiopure $\alpha$-hydroxy esters. Angew. Chem. Int. Ed., 2011, 50, 8387-8390. [87] de Gonzalo, G.; Rodríguez, C.; Rioz-Martínez, A.; Gotor, V. Improvement of the biocatalytic properties of one phenylacetone monooxygenase mutant in hydrophilic organic solvents. Enzyme Microb. Technol., 2012, 50, 43-49.

[88] Cuetos, A.; Lavandera, I.; Gotor, V. Expanding dynamic kinetic protocols: Transaminase-catalyzed synthesis of $\alpha$-substituted $\beta$-amino ester derivatives. Chem. Commun., 2013, 49, 10688-10690.

[89] Limanto, J.; Ashley, E.R.; Yin, J.; Beutner, G.L.; Grau, B.T.; Kassim, A.M.; Kim, M.M.; Klapars, A.; Liu, Z.; Strotman, H.R.; Truppo, M.D. A highly efficient asymmetric synthesis of vernakalant. Org. Lett., 2014, 16, 2716-2719.

[90] Fuchs, C.S.; Hollauf, M.; Meissner, M.; Simon, R.C.; Besset, T.; Reek, J.N.H.; Riethorst, W.; Zepeck, F.; Kroutil, W. Dynamic kinetic resolution of 2-phenylpropanal derivatives to yield $\beta$-chiral primary amines via bioamination. Adv. Synth. Catal., 2014, 356, 2257-2265. 
[91] Peng, Z.; Wong, J.W.; Hansen, E.C.; Puchlopek-Dermenci, A.L.A.; Clarke, H.J. Development of a concise, asymmetric synthesis of a smoothened receptor (SMO) inhibitor: Enzymatic transamination of a 4-piperidinone with dynamic kinetic resolution. Org. Lett., 2014, 16, 860-863.

[92] Chung, C.K.; Bulger, P.G.; Kosjek, B.; Belyk, K.M.; Rivera, N.; Scott, M.E.; Humphrey, G.R.; Limanto, J.; Bachert, D.C.; Emerson, K.M. Process development of C-N cross-coupling and enantioselective biocatalytic reactions for the asymmetric synthesis of niraparib. Org. Process Res. Dev., 2014, 18, 215-227.

[93] Gruber, C.C.; Lavandera, I.; Faber, K.; Kroutil, W. From a racemate to a single enantiomer: Deracemization by stereoinversion. Adv. Synth. Catal., 2006, 348, 1789-1805.

[94] Meena, V.S.; Banoth, L.; Banerjee, U.C. Demonstration of redox potential of Metschnikowia koreensis for stereoinversion of secondary alcohols/1,2-diols. BioMed Res. Int., 2014, doi: $10.1155 / 2014 / 410530$.

[95] Amrutkar, S.M.; Banoth, L.; Banerjee, U.C. One-pot synthesis of (R)-1-(1-naphthyl)ethanol by stereoinversion using Candida parapsilosis. Tetrahedron Lett., 2013, 54, 3274-3277.

[96] Kaliaperumal, T.; Gummadi, S.N.; Chadha, A. Candida parapsilosis ATCC 7330 can also deracemise 1-arylethanols. Biocatal. Biotransform., 2011, 29, 262-270.

[97] Saravanan, T.; Selvakumar, R.; Doble, M.; Chadha, A. Stereochemical preference of Candida parapsilosis ATCC 7330 mediated deracemization: E- versus Z-aryl secondary alcohols. Tetrahedron: Asymmetry, 2012, 23, 1360-1368.

[98] Zhang, R.; Xu, Y.; Xiao, R.; Zhang, B.; Wang, L. Efficient one-step production of (S)-1-phenyl-1,2ethanediol from $(R)$-enantiomer plus $\mathrm{NAD}^{+}-\mathrm{NADPH}$ in-situ regeneration using engineered Escherichia coli. Microb. Cell Fact., 2012, 11, 167.

[99] Fragnelli, M.C.; Hoyos, P.; Romano, D.; Gandolfi, R.; Alcántara, A.R.; Molinari, F. Enantioselective reduction and deracemisation using the non-conventional yeast Pichia glucozyma in water/organic solvent biphasic systems: Preparation of (S)-1,2-diaryl-2-hydroxyethanones (benzoins). Tetrahedron, 2012, 68, 523-528

[100] Xue, Y.-P.; Zheng, Y.-G.; Zhang, Y.-Q.; Sun, J.-L.; Liu, Z.-Q.; Shen, Y.C. One-pot, single-step deracemization of 2-hydroxyacids by tandem biocatalytic oxidation and reduction. Chem. Commun., 2013, 49, 10706-10708.

[101] Voss, C.V.; Gruber, C.C.; Kroutil, W. Deracemisation of secondary alcohols via biocatalytic stereoinversion. Synlett, 2010, 991-998.

[102] Paul, C.E.; Lavandera, I.; Gotor-Fernández, V.; Kroutil, W.; Gotor, V. Escherichia coli/ADH-A: An all-inclusive catalyst for the selective biooxidation and deracemisation of secondary alcohols. ChemCatChem, 2013, 5, 3875-3881.

[103] Kędziora, K.; Díaz-Rodríguez, A.; Lavandera, I.; Gotor-Fernández, V.; Gotor, V. Laccase/TEMPOmediated system for the thermodynamically disfavored oxidation of 2,2-dihalo-1-phenylethanol derivatives. Green Chem., 2014, 16, 2448-2453.

[104] Shin, G.; Mathew, S.; Shon, M.; Kim, B.-G.; Yun, H. One-pot one-step deracemization of amines using $\omega$-transaminases. Chem. Commun., 2013, 49, 8629-8631. 
[105] Chen, Y.; Goldberg, S.L.; Hanson, R.L.; Parker, W.L.; Gill, I.; Tully, T.P.; Montana, M.A.; Goswami, A.; Patel, R.N. Enzymatic preparation of an $(S)$-amino acid from a racemic amino acid. Org. Process Res. Dev., 2011, 15, 241-248.

[106] Seo, Y.-M.; Mathew, S.; Bea, H.-S.; Khang, Y.-H.; Lee, S.-H.; Kim, B.-G.; Yun, H. Deracemization of unnatural amino acid: Homoalanine using D-amino acid oxidase and $\omega$-transaminase. Org. Biomol. Chem., 2012, 10, 2482-2485.

[107] Turner, N.J. Enantioselective oxidation of $\mathrm{C}-\mathrm{O}$ and $\mathrm{C}-\mathrm{N}$ bonds using oxidases. Chem. Rev., 2011, $111,4073-4087$.

[108] Magallanes-Noguera, C.; Ferrari, M.M.; Kurina-Sanz, M.; Orden, A.A. Deracemization of secondary alcohols by chemo-enzymatic sequence with plant cells. J. Biotechnol., 2012, 160, 189-194.

[109] Leisch, H.; Grosse, S.; Iwaki, H.; Hasegawa, Y.; Lau, P.C.K. Cyclohexylamine oxidase as a useful biocatalyst for the kinetic resolution and deracemization of amines. Can. J. Chem., 2012, 90, 39-45.

[110] Li, G.; Ren, J.; Iwaki, H.; Zhang, D.; Hasegawa, Y.; Wu, Q.; Feng, J.; Lau, P.C.K.; Zhu, D. Substrate profiling of cyclohexylamine oxidase and its mutants reveals new biocatalytic potential in deracemization of racemic amines. Appl. Microbiol. Biotechnol., 2014, 98, 1681-1689.

[111] Li, G.; Ren, J.; Yao, P.; Duan, Y.; Zhang, H.; Wu, Q.; Feng, J.; Lau, P.C.K.; Zhu, D. Deracemization of 2-methyl-1,2,3,4-tetrahydroquinoline using mutant cyclohexylamine oxidase obtained by iterative saturation mutagenesis. ACS Catal., 2014, 4, 903-908.

[112] Foulkes, J.M.; Malone, K.J.; Coker, V.S.; Turner, N.J.; Lloyd, J.R. Engineering a biometallic whole cell catalyst for enantioselective deracemization reactions. ACS Catal., 2011, 1, 1589-1594.

[113] Rowles, I.; Malone, K.J.; Etchells, L.L.; Willies, S.C.; Turner, N.J. Directed evolution of the enzyme monoamine oxidase (MAO-N): Highly efficient chemo-enzymatic deracemisation of the alkaloid (士)-crispine A. ChemCatChem, 2012, 4, 1259-1261.

[114] Ghislieri, D.; Green, A.P.; Pontini, M.; Willies, S.C.; Rowles, I.; Frank, A.; Grogan, G.; Turner, N.J. Engineering an enantioselective amine oxidase for the synthesis of pharmaceutical building blocks and alkaloid natural products. J. Am. Chem. Soc., 2013, 135, 10863-10869.

[115] Ghislieri, D.; Houghton, D.; Green, A.P.; Willies, S.C.; Turner, N.J. Monoamine oxidase (MAO-N) catalyzed deracemization of tetrahydro- $\beta$-carbolines: Substrate dependent switch in enantioselectivity. ACS Catal., 2013, 3, 2869-2872.

[116] Köhler, V.; Wilson, Y.M.; Dürrenberger, M.; Ghislieri, D.; Churakova, E.; Quinto, T.; Knörr, L.; Häussinger, D.; Hollmann, F.; Turner, N.J.; Ward, T.R. Synthetic cascades are enabled by combining biocatalysts with artificial metalloenzymes. Nat. Chem., 2013, 5, 93-99.

[117] Schrittwieser, J.H.; Groenendaal, B.; Willies, S.C.; Ghislieri, D.; Rowles, I.; Resch, V.; Sattler, J.H.; Fischereder, E.-M.; Grischek, B.; Lienhart, W.-D.; Turner, N.J.; Kroutil, W. Deracemisation of benzylisoquinoline alkaloids employing monoamine oxidase variants. Catal. Sci. Technol., 2014, 4, 36573664.

[118] Schrittwieser, J.H.; Groenendaal, B.; Resch, V.; Ghislieri, D.; Wallner, S.; Fischereder, E.-M.; Fuchs, E.; Grischek, B.; Sattler, J.H.; Macheroux, P.; Turner, N.J.; Kroutil, W. Deracemization by simultaneous bio-oxidative kinetic resolution and stereoinversion. Angew. Chem. Int. Ed., 2014, 53, 37313734. 
[119] Schrittwieser, J.H.; Resch, V.; Sattler, J.H.; Lienhart, W.-D.; Durchschein, K.; Winkler, A.; Gruber, K.; Macheroux, P.; Kroutil, W. Biocatalytic enantioselective oxidative $\mathrm{C}-\mathrm{C}$ coupling by aerobic $\mathrm{C}-\mathrm{H}$ activation. Angew. Chem. Int. Ed., 2011, 50, 1068-1071.

[120] Yasukawa, K.; Nakano, S.; Asano, Y. Tailoring D-amino acid oxidase from the pig kidney to $R$ stereoselective amine oxidase and its use in the deracemization of $\alpha$-methylbenzylamine. Angew. Chem. Int. Ed., 2014, 53, 4428-4431.

[121] Tudorache, M.; Nica, S.; Bartha, E.; Lupan, I.; Parvulescu, V.I. Sequential deracemization of sulfoxides via whole-cell resolution and heterogeneous oxidation. Appl. Catal. A-Gen., 2012, 441-442, $42-46$.

[122] Wallner, S.R.; Pogorevc, M.; Trauthwein, H.; Faber, K. Biocatalytic enantioconvergent preparation of sec-alcohols using sulfatases. Eng. Life Sci., 2004, 4, 512-516.

[123] Szymański, W.; Westerbeek, A.; Janssen, D.B.; Feringa, B.L. A simple enantioconvergent and chemoenzymatic synthesis of optically active $\alpha$-substituted amides. Angew. Chem. Int. Ed., 2011, 50, 10712-10715.

[124] Schober, M.; Faber, K. Inverting hydrolases and their use in enantioconvergent biotransformations. Trends Biotechnol., 2013, 31, 468-478.

[125] Schober, M.; Gadler, P.; Knaus, T.; Kayer, H.; Birner-Grünberger, R.; Gülly, C.; Macheroux, P.; Wagner, U.; Faber, K. A stereoselective inverting sec-alkylsulfatase for the deracemization of secalcohols. Org. Lett., 2011, 13, 4296-4299.

[126] Schober, M.; Knaus, T.; Toesch, M.; Macheroux, P.; Wagner, U.; Faber, K. The substrate spectrum of the inverting sec-alkylsulfatase Pisa1. Adv. Synth. Catal., 2012, 354, 1737-1742.

[127] Fuchs, M.; Toesch, M.; Schober, M.; Wuensch, C.; Faber, K. Chemoenzymatic asymmetric total synthesis of $(R)$-lasiodiplodin methyl ether through a sulfatase-based deracemization process. Eur. J. Org. Chem., 2013, 356-361.

[128] Schober, M.; Toesch, M.; Knaus, T.; Strohmeier, G.A.; van Loo, B.; Fuchs, M.; Hollfelder, F.; Macheroux, P.; Faber, K. One-pot deracemization of sec-alcohols: Enantioconvergent enzymatic hydrolysis of alkyl sulfates using stereocomplementary sulfatases. Angew. Chem. Int. Ed., 2013, 52, 3277-3279.

[129] Kotik, M.; Archelas, A.; Faměrová, V.; Oubrechtová, P.; Křen, V. Laboratory evolution of an epoxide hydrolase - Towards an enantioconvergent biocatalyst. J. Biotechnol., 2011, 156, 1-10.

[130] Kotik, M.; Zhao, W.; Iacazio, G.; Archelas, A. Directed evolution of metagenome-derived epoxide hydrolase for improved enantioselectivity and enantioconvergence. J. Mol. Catal. B: Enzym., 2013, 91, 44-51.

[131] Min, J.Y.; Lee, E.Y. Biosynthesis of $(R)$-1,2-phenylethanediol and (R)-4-chloro-1,2phenylethanediol by using two recombinant cells expressing enantiocomplementary epoxide hydrolases. J. Ind. Eng. Chem., 2012, 18, 160-164.

[132] Matsumoto, K.; Usuda, K.; Okabe, H.; Hashimoto, M.; Shimada, Y. Synthesis of optically active heterocyclic compounds via deracemization of 1,2-diol monotosylate derivatives bearing a long aliphatic chain by a combination of enzymatic hydrolysis with Mitsunobu inversion. Tetrahedron: Asymmetry, 2013, 24, 108-115. 
[133] Houiene, Z.; Merabet-Khelassi, M.; Bouzemi, N.; Riant, O.; Aribi-Zouioueche, L. A green route to enantioenriched $(S)$-arylalkyl carbinols by deracemization via combined lipase alkalinehydrolysis/Mitsunobu esterification. Tetrahedron: Asymmetry, 2013, 24, 290-296. 


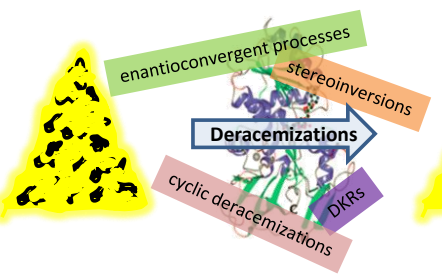

Why leave a job half done? Recent progress in enzymatic deracemizations

\section{Alba Díaz-Rodríguez, Iván Lavandera, and Vicente Gotor}

Departamento de Química Orgánica e Inorgánica, Instituto Universitario de Biotecnología de Asturias, University of Oviedo, C/ Julián Clavería 8, 33006 Oviedo, Spain

Recent examples of enzymatic deracemizations to obtain enantioenriched compounds in high yields will be reviewed herein 